\title{
Comparing Bacterial Diversity in Two Full-Scale Enhanced Biological Phosphate Removal Reactors Using 16S Amplicon Pyrosequencing
}

\author{
Ilunga Kamika*, Shohreh Azizi, Memory Tekere \\ Department of Environmental Sciences, University of South Africa, \\ PO Box 392 UNISA 0003
}

Received: 28 November 2016

Accepted: 16 February 2017

\begin{abstract}
Despite their stability and the widespread use of enhanced biological phosphorus removal (EBPR), little is known about their microbial composition and activity. In our study we investigated highthroughput pyrosequencing of bacterial communities from two full-scale EBPR reactors of South Africa. Findings indicated that both EBPRs harboured high bacterial similarity, ranging from 83 to $100 \%$ with a diverse community dominated by Proteobacteria (57.04 to $79.48 \%$ for failed EBPR and 61.7 to $85.39 \%$ for successful EBPR) throughout the five selected treatment zones with the exception of the fermenter (Bacteroidetes: 55.84\%) from the successful EBPR. However, a lower dissimilarity was observed with the presence of 70 unique bacterial genera from successful EBPRs belonging to Gammaproteobacteria, Betaproteobacteria, and Actinobacteria, while 69 unique genera from failed EBPR belonged to Alphaproteobacteria, Betaproteobacteria, and Clostridia. The failed EBPR (54.58\%) revealed less fermenting bacteria in the fermenter as compared to the successful EBPR (73.58\%). More detrimental organisms and less nitrifying/denitrifying bacteria were also found in failed EBPR than in the successful EBPR, as well as phosphate-accumulating bacteria. Canonical correspondence analysis (CCA) displayed a very low relationship between microbial patterns, $\mathrm{pH}$ and $\mathrm{DO}$ - suggesting that these environmental factors played a major role in community dissimilarity. Aerobic zones appeared to have the highest dissimilarity between both EBPRs, with the failed EBPR predominated by Acidovorax (26.2\%) and the successful EBPR with unclassified Rhodocyclaceae (37.24\%). Furthermore, 21.47\% of readings (failed EBPR) and $17.18 \%$ of readings (successful EBPR) could not be assigned to taxonomic classifications, highlighting the high diversity level of novel microbial species in such an environment.
\end{abstract}

Keywords: EBPR, microbial diversity, aerobic zone, anaerobic zone, pyrosequencing

*e-mail:kamiki@unisa.ac.za, alainkamika2@yahoo.com 


\section{Background}

Pollution of water sources is currently a global concern due to the detrimental effects of pollutants on both human and animal health [1]. In South Africa, like in many other developing countries, there is a problem with an influx of population into urban areas. This together with the growth of major cities and industrial activities has led to an increase in the municipal biomass waste in effluents being discharged into the environment with the contamination of water sources. Moreover, the volume of wastes that goes into wastewater treatment plants is enormous, while the treatment efficiency is hampered by a number of factors such as high capital and operating costs, which render some technologies unfeasible primarily for application in rural areas [2].

To deal with this issue, several technologies have been developed and these include chemical precipitation, chemical oxidation, ion exchange, etc. Due to their advantages over the conventional methods, biological treatment methods such as enhanced biological phosphorus removal (EBPR) have been widely seen as the most effective and eco-friendly option of the available treatment processes of wastewater [3]. This process requires the presence of diverse microorganisms to remove organic contents and nutrients as well as other toxic materials from domestic and industrial wastewater and to protect receiving water bodies from eutrophication [4-5]. Although the EBPR process is regarded as one of the most successful for the removal of phosphorus from wastewater, the phosphorus removal capacity can also be prone to apparent instability and unreliability, and deterioration is often attributed to detrimental bacteria present in the system [4].

More than three decades ago, researchers made the first attempts to identify these microorganisms involved in EBPR based on culture-dependent techniques [6-7]. It has then been reported that the naturally occurring polyphosphate-accumulating organisms (PAOs) were responsible for phosphorus removal, whereas the presence of glycogen-accumulating organisms (GAOs) in the EBPR process was the main cause of EBPR deterioration as the latter also compete for volatile fatty acid (VFA) as a substrate for energy [5]. However, the advent of molecular tools showed that several pure cultures of PAOs such as Acinetobacter, Microlunatus, Tetrasphaera, and Lampropedia genera were not having great effect in full-scale wastewater treatment plants (WWTPs), and a high diversity of phylogenetic groups involved in the removal of phosphorus were present in EBPR sludge [8]. Furthermore, PAOs such as Candidatus Accumulibacter phosphatis and the actinobacterial genus Tetrasphaera that enable enhanced biological phosphorus removal in full-scale WWTPs have been identified and characterized [7, 9].

While it remains a challenge to identify and isolate the PAOs or GAOs in pure cultures using alternative molecular analyses, metagenomic analysis using nextgeneration sequencing appears to be more successful for characterizing the microbial ecology of the EBPR systems as it offers the possibility of studying the diversity of phylogenetic groups and the functional potential of a complex community [10]. Furthermore, their success in profiling microbial communities in a wider range has been used as a blueprint by which more studies can be designed and comprehensive hypotheses generated [11]. Recently, very few metagenomic studies using next-generation sequencing have been carried out on metagenomes from a full-scale EBPR [4, 10, 1213]. In addition, very few studies have investigated the microbial community throughout all treatment zones of the EBPR. Since each EBPR appeared to be unique, this study investigated for the first time the microbial community of the targeted full-scale EBPRs using a pyrosequencing platform. The microbial diversity of the two full-scale EBPRs (Olifantsvlei Wastewater Treatment Works and the Bushkoppies Wastewater Treatment Works) was compared in order to provide a more detailed understanding of their relationships to operational variables and design in complex habitats. Despite the fact that Olifantsvlei Wastewater Treatment Works and the Bushkoppies Wastewater Treatment Works have been similarly designed with the use of activated sludge, BNR, extended aeration, and maturation ponds (except for the absence of a pre-anoxic zone at the latter, and also since these plants receive the same type of influents), the Bushkoppies Wastewater Treatment works showed consistent noncompliance as compared to the Olifantsvlei Wastewater Treatment Works.

\section{Materials and Methods}

\section{Study Area and Wastewater Sample Collection}

Wastewater samples were collected in April 2015 from two treatment plants, namely at the Olifantsvlei Wastewater Treatment Works (26.321864S, 27.901647E) and the Bushkoppies Wastewater Treatment Works (26.311111S, 27.935E) in Johannesburg. A comparison of these two treatment works was performed in order to assess the consistent noncompliance of the latter. These EBPRs use activated sludge, BNR, extended aeration, and maturation ponds, and receive the same type of influents and are subjected to the same climatic conditions and are configured as Johannesburg and anaerobic-oxic process reactors and combined in the secondary clarifier. The fermenter responsible for fermenting organic matter for producing VFA is a combined system that recycled the sludge back to each train (Fig. 1). Due to the above and for the sake of this study, the targeted plants were referred to as "failed unit" for Bushkoppies and "successful unit" for Olifantsvlei. Samples of $1 \mathrm{~L}$ were collected in sterile plastic sampling bottles in triplicate from the primary settling tank (PST), fermenter, anaerobic zone, anoxic zone, and aerobic zone, and immediately placed in a cooler box $\left(4^{\circ} \mathrm{C}\right)$ for transportation to the laboratory for physicochemical analyses and microbial diversity 


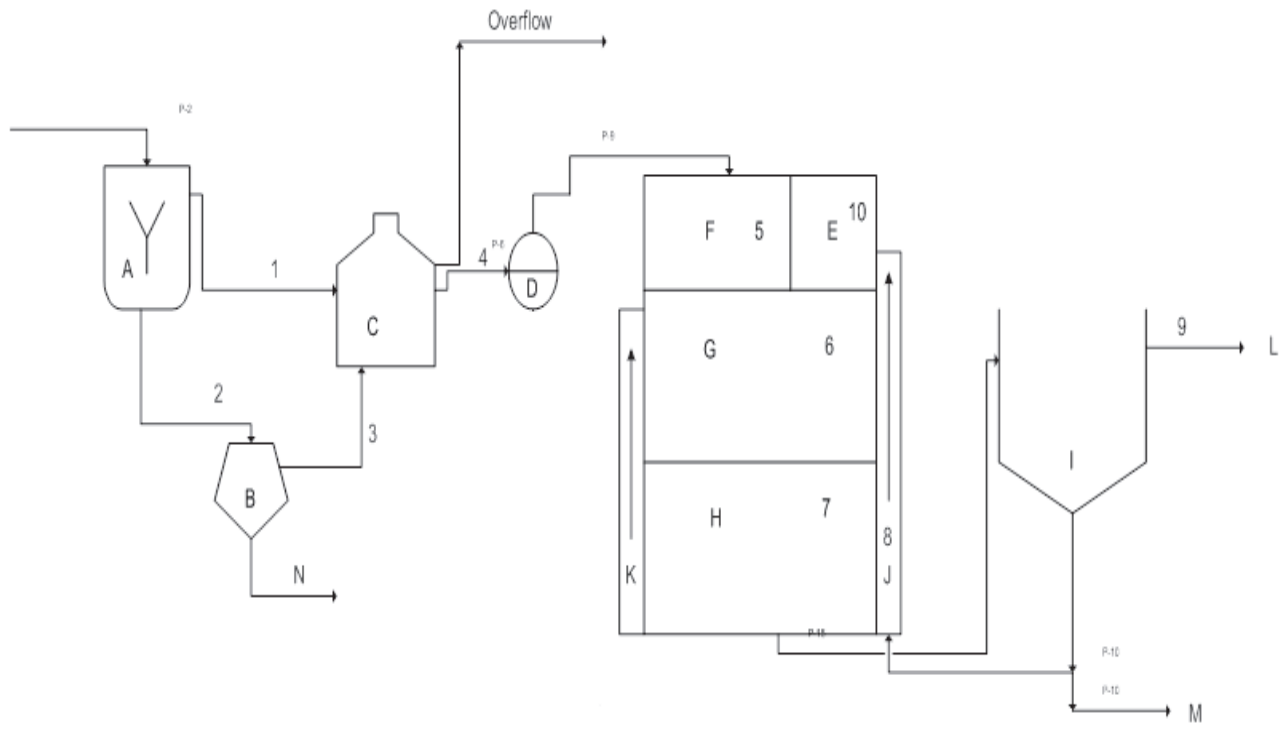

Fig. 1. Schematic design of the EBPR.

Legend: Successful EBPR

A: Primary settling tanks ( $2 \mathrm{x})$; B: Fermenter ( 2 tanks). Sludge from PST is pumped into one tank. Remains for $4-6$ days. Waste out with settled sewerage from PST. Other tank is then filled up.; C: Balancing tank; D: Division box, out flow from balancing tank.; E: Preanoxic. Receives the RAS; F: Anaerobic zone. Receives outflow from balancing tank.; G: Anoxic zone. Receives recycle from aerobic zone; H: Aerobic zone; I: Secondary settling tank. Suction lift tanks (3 x). Difficult to control.; J: Return activated sludge (RAS) from the bottom of the SST's; K: Recycle stream from aerobic to anoxic.; L: Water effluent from SST goes to disinfection and river; M: Waste activated sludge. Wasted directly from aeration tank.; N: Anaerobic digestion.

Failed EBPR:

B: Fermenter, Receives sludge from the PST. Kept it settle for 4 days. E: Pre-anoxic not present, F: Anaerobic zone. Excessive "bubbling" where the water enters the zone.; I: Secondary settling tank. Mechanical scrapped tanks. Removes sludge better.

studies. No specific permit was needed for collecting the wastewater samples in the described sample area, and this study did not involve endangered or protected species. However, responsible officers with informed consent assisted with the collection of wastewater samples. In order to assess environmental variables, samples were homogenously mixed and filtered using No. 1 filter paper (Whatman). The filtered samples were then used to determine chemical oxygen demand (COD), nitrate, and phosphate. To test for $\mathrm{NO}_{3}^{-1}$, the sodium salicylate method was used as reported by Monteiro et al. [14]. Briefly, $50 \mathrm{ml}$ of the samples were pippeted into a $\mathrm{ml}$ beaker and mixed with $1 \mathrm{ml}$ of the salicylate solution. The mixture was dried in an oven at $105^{\circ} \mathrm{C}$ to allow the formation of $\mathrm{NO}_{2}^{+1}$ from $\mathrm{NO}_{3}^{-1}$. Then $1 \mathrm{ml}$ of sulfuric acid was added and allowed to cool for $10 \mathrm{~min}$, and $7 \mathrm{ml}$ of the solution containing sodium hydroxide and sodium and potassium tartrate were later added. The solution was later made up with water and analyzed in a spectrophometer [14]. For $\mathrm{PO}_{4}^{-3}$ we used the $424 \mathrm{f}$ standard method as reported by APHA [15]. 424f uses ammonium molybdate and potassium antimonyl tartrate in order to react in an acidic medium with orthophosphate to form a heteropoly acid (phosphomolybdic acid) that is reduced to intensely coloured molybdenum blue by ascorbic acid. The closed reflux method was also used to measure COD concentration [15], whereas $\mathrm{pH}, \mathrm{DO}$, electrical conductivity (EC), and temperature were measured onsite using specific probes (HACH, Germany).
Metagenomic DNA Extraction, Amplification, and Pyrosequencing of Bacterial 16S RNA Genes

For microbial analysis, unfiltered samples were homogenously mixed and a $50 \mathrm{~mL}$ aliquot was taken and centrifuged at $10,000 \mathrm{x} \mathrm{g}$ for five minutes at $4^{\circ} \mathrm{C}$. The harvested cell pellets were re-suspended in 1x TE buffer (pH 8.0). The suspensions were well mixed and microbial DNA was extracted with the ZR Fungal/Bacterial DNA $\mathrm{Kit}^{\mathrm{TM}}$ (Zymo Research Corporation, USA) according to the procedures provided by the manufacturer. In order to monitor/assess for any contamination, a series of negative controls containing no DNA templates was also used throughout this experiment. The integrity and purity of the metagenomic DNA was later assessed on the $0.8 \%$ agarose gel and NanoDrop ${ }^{\mathrm{TM}}$ spectrophotometer (NanoDrop $^{\mathrm{TM}}$ 2000, Thermo Scientific, Japan). The PCR reaction was performed on all the extracted metagenomic DNA samples as well as negative controls using the universal primer $27 \mathrm{~F}$ and 518R [5]. This primer pair amplifies approximately the $500 \mathrm{bp}$ of $16 \mathrm{~S}$ rRNA gene sequence targeting variable region $\mathrm{V} 1$ to $\mathrm{V} 3$ of the 16S rRNA gene. Each PCR reaction contained 25 $\mu \mathrm{L}$ of $2 \mathrm{X}$ DreamTaq Green Master Mix (DNA polymerase, dNTPs and $4 \mathrm{mM} \mathrm{MgCl} 2), 22 \mu \mathrm{L}$ of nucleasefree water, $1 \mu \mathrm{L}$ of forward primer $(0.2 \mu \mathrm{M}), 1 \mu \mathrm{L}$ of reverse primer $(0.2 \mu \mathrm{M})$, and $1 \mu \mathrm{L}$ of metagenomic DNA (50-100 ng $\left.\mu \mathrm{L}^{-1}\right)$ to make up a volume of $50 \mu \mathrm{L}$. 
All PCR reactions were performed on ice to minimize non-specific amplification and primer dimerization. The PCR cycle started with an initial denaturation step at $94^{\circ} \mathrm{C}$ for five minutes, followed by 30 cycles of denaturation at $94^{\circ} \mathrm{C}$ for one minute, annealing at $55^{\circ} \mathrm{C}$ for 30 seconds and extension at $72^{\circ} \mathrm{C}$ for one minute and 30 seconds, and a final extension at $72^{\circ} \mathrm{C}$ for 10 minutes, followed by cooling to $4^{\circ} \mathrm{C}$. The PCR product $(10 \mu \mathrm{L})$ was loaded in $1 \%$ $(\mathrm{m} / \mathrm{v})$ agarose gel (Merck, South Africa) stained with 5\% of $10 \mathrm{mg} \mathrm{mL}^{-1}$ ethidium bromide (Merck, South Africa) and visualised under an ultraviolet transilluminator (InGenius Bio Imaging System, Syngene, Cambridge, UK). The correct PCR amplicons were excised and purified using the DNA clean and concentrator kits (Zymo Research Corporation, USA). The purified DNA samples were then quantified using a NanoDrop spectrophotometer. The used primers contained the appropriate adaptor and barcode sequences necessary for running the samples on the GS-FLX-Titanium (Roche). Prior to the paired-end pyrosequencing services provided by Inqaba Biotechnology Industries (South Africa) using the GS-FLX-Titanium series (Roche, Switzerland), replicates for respective samples were pooled together at approximately equimolar concentrations based on the library concentrations and calculated amplicon sizes.

\section{Processing of Pyrosequencing Data and Statistical Analysis}

The raw sequences generated from pyrosequencing were processed in a Mothur pipeline for quality control (QC) to remove artificial replicate sequences produced by sequencing artifacts (primers, barcodes, adaptor sequences, etc.) and low-quality sequences [16]. Sequences that passed QC were further pre-screened for identification of ribosomal readings (at least $80 \%$ identity) and removal of non-ribosomal readings using qiimeuclust and the following RNA databases: Greengenes, LSU, SSU, and RDP. Moreover, chimeric sequences were identified from ribosomal sequences and removed using UCHIME according to the de novo method [17]. The nonchimeric rRNA sequences obtained were analyzed using the Ribosomal Database Project (RDP) Classifier tool of RDP pyrosequencing pipeline [18] and sequences were classified with a $97 \%$ confidence threshold. These metasequences were later aligned using the RDP align tool in the same pipeline, and the cluster files were generated for each sample with the RDP complete linkage clustering tool. Furthermore, the rarefaction curves were created by using the RDP rarefaction tool. Genetic distance was determined and sequences clustered into operational taxonomic units (OTUs) at 3\% genetic distance using the nearest-neighbour method, and a representative sequence was chosen for each cluster/OTU at each distance. The choice of nucleotide cutoff in this study was based on Brown et al. [12], who reported that the bacterial diversity could decrease with an increase of cutoff. Furthermore, the default mismatch cutoff at $3 \%$ for cdhit- 454 was also set in order to recover approximately $95 \%$ of readings.
Since increasing the cutoff strongly affects the overall number of readings assigned to a specific taxon, in this study the default genetic distance cutoff of 3\% was used. The Shanon diversity index and the Chaol richness estimator were calculated for each sample. The relative abundance (\%) of individual taxa within each community was calculated by comparing the number of sequences assigned to a specific taxon against the number of total sequences obtained for that sample. The similarity and dissimilarity in bacterial community structure within both wastewater treatment plants was analyzed using the Jaccard-Sørensen index [18]. Statistically, we should indicate that both indexes can be shown as similarity or dissimilarity indexes. Canonical correspondence analysis (CCA) for the environmental parameters was also performed to explore the interrelationships between them and their possible effect on a bacterial community. PAST 2.06 statistical software was used to generate CCA bi-plots [19]. Generated data was later made publicly available at the DDBJ Sequence Read Archive (DRA) under the accession number PRJDB4407.

\section{Results and Discussion}

\section{Sequencing}

The enhanced biological phosphorus removal processes have been seen widely as the most reliable approach for the treatment of wastewater generated from both municipal and industrial activities [20]. Among its microbial population, bacteria have been reported to play the most essential and critical role for the removal of pollutants [21]. In the present study, the bacterial community structure and species richness, from five zones of two wastewater treatment plants situated in Johannesburg, were analyzed using pyrosequencing of the 16S rRNA gene. Extracted rDNA was found to be of high quality, with A260/280 ratios of approximately 1.8 for all samples. As activated sludge samples are seen as complex samples due to the presence of several inhibitors, the purity, integrity, and size of the rDNA from such a matrix are critical and play a major role in the specific yield (rDNA/g sample) of rDNA by later impacting the accuracy of the results of metagenomic studies, especially in high-diversity communities [21-22]. When investigating the microbial community of a full-scale EBPR, Albertsen et al. [4] revealed that some microbial phyla such as Actinobacteria (Tetrasphaera) and Chloroflexi have been underrepresented from the metagenome datasets due to the DNA extraction bias.

Pyrosequencing of DNA amplicons from high-quality rDNA generated a total range of 1,120 to 2,436 readings from treatment zones (aerobic, anoxic, anaerobic, fermenter, and PST) with Anoxic A (2,030 readings) and Anaerobic B $(2,436$ readings $)$ having the highest readings from both failed and successful EBPRs, respectively (Table 1). 
Table 1. Sequencing statistics from wastewater samples.

\begin{tabular}{|c|c|c|c|c|c|c|c|c|c|}
\hline \multirow[t]{2}{*}{ WWTWs } & \multirow[t]{2}{*}{ Sampling zone } & \multirow{2}{*}{$\begin{array}{l}\text { No. of } \\
\text { seq. }\end{array}$} & \multirow{2}{*}{$\begin{array}{c}\text { Average } \\
\text { length (bp) }\end{array}$} & \multicolumn{2}{|c|}{ QC passed } & \multicolumn{2}{|c|}{ rRNA seq } & \multicolumn{2}{|c|}{$\begin{array}{l}\text { No. of non- } \\
\text { chimeric seq. }\end{array}$} \\
\hline & & & & No. & $\%$ & No. & $\%$ & No. & $\%$ \\
\hline \multirow{5}{*}{ Failed EBPR } & Aerobic A & 1,950 & $270.59 \pm 158.18$ & 1,433 & 73.49 & 1,180 & 60.51 & 1,152 & 59.08 \\
\hline & Anoxic A & 2,030 & $329.17 \pm 188.14$ & 1,807 & 89.01 & 1,530 & 75.37 & 1,227 & 60.44 \\
\hline & Anaerobic A & 1,908 & $339.27 \pm 186.92$ & 1,658 & 86.9 & 1,420 & 74.42 & 1,118 & 58.60 \\
\hline & Fermenter A & 1,705 & $341.84 \pm 192.90$ & 1,544 & 90.56 & 1,375 & 80.65 & 1,121 & 65.75 \\
\hline & PSTA & 1,120 & $274.19 \pm 152.80$ & 872 & 77.86 & 823 & 73.48 & 736 & 65.71 \\
\hline \multirow{5}{*}{$\begin{array}{l}\text { Successful } \\
\text { EBPR }\end{array}$} & Aerobic B & 1,750 & $318.32 \pm 187.47$ & 1,568 & 89.6 & 1,360 & 77.71 & 1,217 & 69.54 \\
\hline & Anoxic B & 1,876 & $316.83 \pm 185.12$ & 1,682 & 89.66 & 1,523 & 81.18 & 1,166 & 62.15 \\
\hline & Anaerobic B & 2,436 & $303.63 \pm 181.97$ & 2,203 & 90.44 & 1,790 & 73.48 & 1,556 & 63.88 \\
\hline & Fermenter B & 1,748 & $366.81 \pm 187.09$ & 1,610 & 92.11 & 1,496 & 85.58 & 1,217 & 69.62 \\
\hline & PSTB & 1,269 & $272.70 \pm 162.78$ & 978 & 77.07 & 976 & 76.91 & 810 & 63.83 \\
\hline
\end{tabular}

Note: All percentages of sequences refer to the total sequences of each sampling site.

Of the obtained readings, $76.17-90.56 \%$ were for failed the EBPR and 77.07-92.11\% were for the successful EBPR and were able to meet the quality criteria (QC). Sequences up to $80.65 \%$ from the failed EBPR and $85.58 \%$ from the successful EBPR were found to be of ribosomal origin. The ribosomal sequences were then analyzed for the presence of chimeric sequences, and 520-1,227 and 810-1,556 high-quality non-chimeric rRNA readings
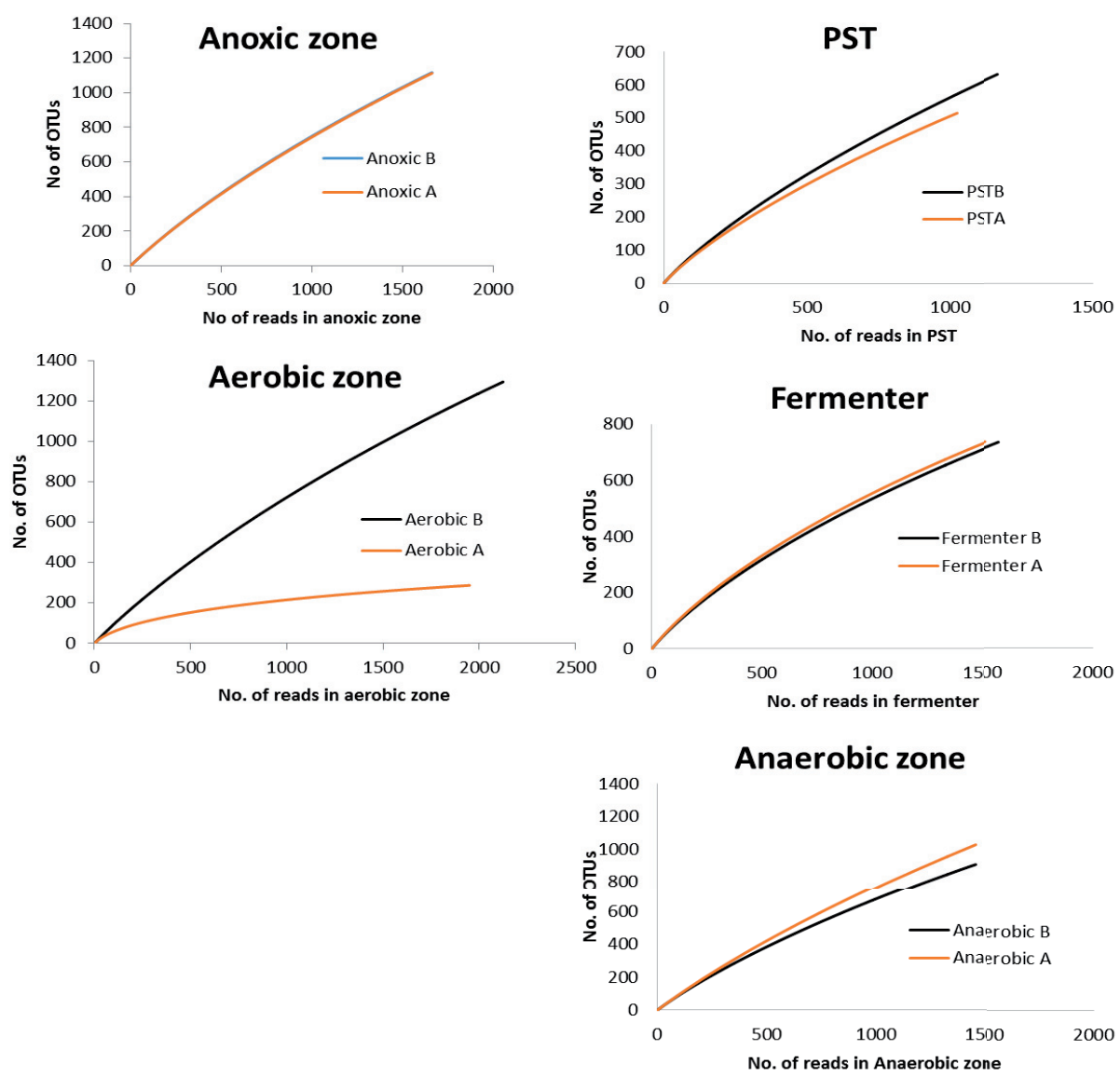

Fig. 2. Rarefaction curves dissimilarity levels at failed EBPR (Aerobic A, Anoxic A, Anaerobic A, Fermenter A, and PSTA) and successful EBPR (Aerobic B, Anoxic B, Anaerobic B, Fermenter B, and PSTB) 
Table 2. Diversity indices from 10 sampling points of two wastewater treatment works.

\begin{tabular}{|c|c|c|c|c|}
\hline & Samples & OTU & $\begin{array}{c}\text { Shannon } \\
\text { index }\end{array}$ & Chao1 \\
\hline \multirow{4}{*}{$\begin{array}{c}\text { Failed } \\
\text { EBPR }\end{array}$} & Aerobic A & 287 & 5.36 & $1,025.14$ \\
\cline { 2 - 5 } & Anoxic A & 1,216 & 6.84 & $3,526.05$ \\
\cline { 2 - 5 } & Anaerobic A & 1,195 & 6.86 & $3,871.12$ \\
\cline { 2 - 5 } & Fermenter A & 737 & 6.12 & $1,672.36$ \\
\cline { 2 - 5 } & PSTA & 516 & 5.64 & $1,533.9$ \\
\hline \multirow{4}{*}{$\begin{array}{c}\text { Successful } \\
\text { EBPR }\end{array}$} & Aerobic B & 1,296 & 6.83 & $3,496.38$ \\
\cline { 2 - 5 } & Anoxic B & 1,118 & 6.8 & $3,519.88$ \\
\cline { 2 - 5 } & Anaerobic B & 907 & 6.48 & $2,661.09$ \\
\cline { 2 - 5 } & Fermenter B & 735 & 5.95 & $1,749.55$ \\
\cline { 2 - 5 } & PSTB & 633 & 5.94 & $1,965.66$ \\
\hline
\end{tabular}

from failed and successful EBPRs respectively were obtained and considered for further analysis. All nonchimeric readings of an average between $270.59 \pm 158.18$ bp and $366.81 \pm 187.09 \mathrm{bp}$ were successfully classified at the domain level. Previous studies further revealed that the accurate classification of $16 \mathrm{~S}$ rDNA sequences to the perceived diversity of the microbial community is strongly dependent on the high quality of the $16 \mathrm{~S}$ database [11, 23], which in return can be influenced by sample preparation, primer selection, and the formation of chimeric $16 \mathrm{~S}$ amplification products [24].

\section{Diversity Indices and Community Species Richness}

During the last decade, studies on diversity level have reported the potential positive correlation between biodiversity and micro-ecosystem function [25]. It has also been stated that the stability of a micro-ecosystem function increases as biodiversity increases [26]. According to Saikaly et al. [27], minimum species richness is needed in order to maintain ecosystem function in a particular environment, while a large microbial diversity and species richness is required in order to maintain a stable micro-ecosystem in a varying environment. In this study, the RDP pyrosequencing pipeline was used to determine a bacterial community from two EBPRs by assigning the read tags to different operational taxonomic units (OTUs) at 3\% nucleotide cutoff. As the number of OTUs gives an approximation of species diversity in a sample [28], a total of 8,640 OTUs were recovered from the 10 sampling points. It was noted that the successful wastewater treatment plant had the highest OTUs of 1,296 at an aerobic zone (aerobic B). Regardless of the fact that the successful EBPR was having more OTUs and readings than the failed EBPR, the rarefaction analysis has revealed no significant difference in the number of readings and OTUs at the same sampling zone of both EBPRs. This indicates a similar level of diversity with the exception of the aerobic zone, where the level of bacterial diversity was apparently not similar. Furthermore, the rarefaction curves showing the plot of number of readings versus the number of OTUs is illustrated in Fig. 2.

In order to ascertain microbial complexity within each sampling point, the Shannon-Weaver index and Chaol richness estimator were determined at 3\% cutoff (Table 2). The Shannon-Weaver diversity index ranges from 0 (or $0 \%$ ), indicating pronounced dominance, to 1 (or $100 \%$ ), indicating equal abundance of all species [29]. In the present study, the diversity index (Shannon) revealed a very close level of diversity between both wastewater treatment plants with 30.82 for failed EBPR and 32 for successful EBPR. Furthermore, the diversity index within individual sampling points appeared not to

Table S1 Pairwise bacterial community similarity between the sampling points using Jaccard index (light grey) and Sørensen index (dark grey) at 3\% nucleotide cutoff level

\begin{tabular}{|c|c|c|c|c|c|c|c|c|c|c|}
\hline & $\begin{array}{c}\text { Aerobic } \\
\text { A }\end{array}$ & $\begin{array}{c}\text { Aerobic } \\
\text { B }\end{array}$ & $\begin{array}{c}\text { Anaerobic } \\
\text { A A }\end{array}$ & $\begin{array}{c}\text { Anaerobic } \\
\text { _B }\end{array}$ & $\begin{array}{c}\text { Anoxic } \\
\text { A }\end{array}$ & $\begin{array}{c}\text { Anoxic } \\
\text { B }\end{array}$ & $\begin{array}{c}\text { Fermenter } \\
\text { A A }\end{array}$ & $\begin{array}{c}\text { Fermenter } \\
\text { B }\end{array}$ & PST_A & PST_B \\
\hline Aerobic_A & 0.00 & 96 & 66 & 99 & 93 & 98 & 97 & 100 & 94 & 96 \\
\hline Aerobic_B & 98 & 0.00 & 94 & 86 & 90 & 84 & 99 & 99 & 94 & 48 \\
\hline Anaerobic_A & 80 & 97 & 0.00 & 91 & 83 & 86 & 98 & 99 & 95 & 98 \\
\hline Anaerobic_B & 99 & 93 & 95 & 0.00 & 95 & 74 & 99 & 100 & 93 & 98 \\
\hline Anoxic_A & 96 & 95 & 90 & 97 & 0.00 & 83 & 98 & 98 & 95 & 99 \\
\hline Anoxic_B & 99 & 91 & 92 & 85 & 91 & 0.00 & 98 & 99 & 100 & 95 \\
\hline Fermenter_A & 98 & 100 & 99 & 100 & 99 & 99 & 0.00 & 91 & 98 & 99 \\
\hline Fermenter_B & 100 & 100 & 100 & 100 & 99 & 99 & 95 & 0.00 & 95 & 99 \\
\hline PST_A & 97 & 97 & 97 & 97 & 97 & 100 & 99 & 98 & 0.00 & 95 \\
\hline PST_B & 98 & 65 & 99 & 99 & 99 & 98 & 100 & 100 & 97 & 0.00 \\
\hline
\end{tabular}

Note: values are expressed in percentage (\%) 


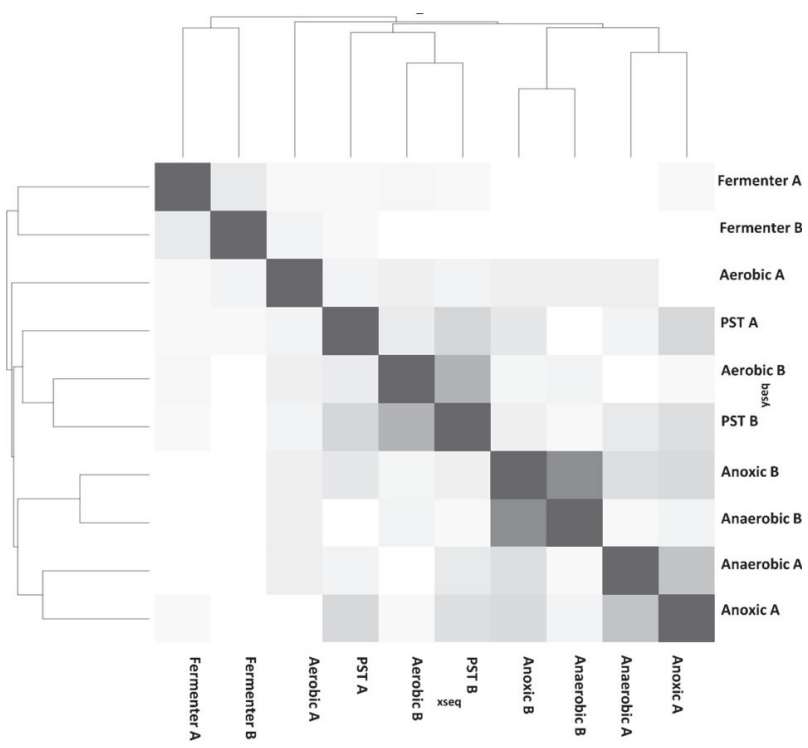

Fig. 3. Heat map generated from Sørensen index highlighting the similarity at $3 \%$ cutoff level among the 10 samples collected from both EBPR treatment plants. The higher the colour intensity, the higher the bacterial similarity between the pair. be significantly different ( $p>0.05$ ) within both successful and failed EBPRs.

However, the species richness from both plants was also determined using the Chaol estimator at 3\% nucleotide cutoff. Similar to the Shannon index, the observed and estimated richness index indicated higher bacterial richness at 3\% cutoff at the successful EBPR when compared to the failed EBPR. The Chaol richness estimator revealed a species richness ranging between $1,025.14$ and 3,871.12, and 1,749.55 and 3,519.88 from failed and successful EBPR, respectively. In the failed wastewater treatment plant, the anaerobic zone appeared to have the highest bacterial species richness, whereas in the successful wastewater treatment plant, the anoxic zone had the highest bacterial species richness. The pairwise bacterial community similarity between the sampling points of each wastewater treatment plant was confirmed using the Jaccard and Sørensen index based on the presence and absence of each OTU (Table S1). The Jaccard and Sørensen index revealed no or slightly similar levels of diversity between each pair of the same sampling points and between both plants.
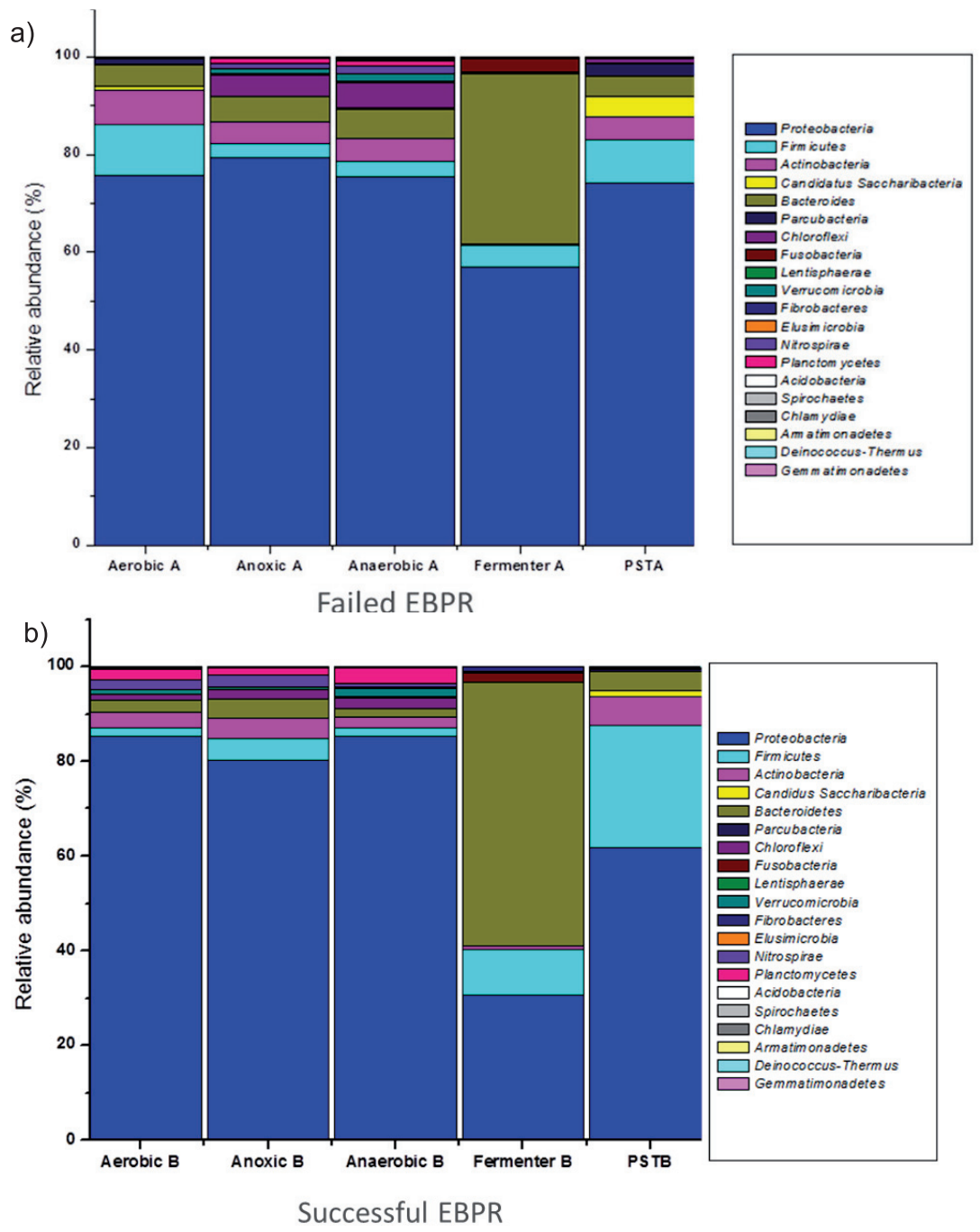

Fig. 4. Taxonomic distribution of different bacterial phyla in both treatment plants from the Johannesburg area: a) the zone belonging to failed EBPR and $b$ ) the zone belonging to the successful EBPR. 
Table S2. Unifrac metric $\mathrm{p}$ values.

\begin{tabular}{|c|c|c|c|c|c|c|c|c|c|c|}
\hline & $\begin{array}{c}\text { Aerobic } \\
\text { AA }\end{array}$ & $\begin{array}{c}\text { Aerobic } \\
\text { _B }\end{array}$ & $\begin{array}{c}\text { Anaerobic } \\
\text { _A }\end{array}$ & $\begin{array}{c}\text { Anaerobic } \\
\text { B }\end{array}$ & $\begin{array}{c}\text { Anoxic } \\
\text { A }\end{array}$ & $\begin{array}{c}\text { Anoxic } \\
\text { B }\end{array}$ & $\begin{array}{c}\text { Fermenter } \\
\text { A }\end{array}$ & $\begin{array}{c}\text { Fermenter } \\
\text { B }\end{array}$ & PST_A & PST_B \\
\hline Aerobic_A & & 0.02 & 0.09 & 0.05 & 0.04 & 0.01 & $\mathbf{0 . 0 0}$ & 0.02 & 0.05 & 0.01 \\
\hline Aerobic_B & 0.02 & & 0.04 & 0.01 & 0.30 & 0.03 & 0.03 & 0.03 & 0.01 & 0.07 \\
\hline Anaerobic_A & 0.09 & 0.04 & & 0.01 & 0.02 & 0.02 & 0.03 & 0.02 & 0.01 & 0.03 \\
\hline Anaerobic_B & 0.05 & 0.01 & 0.01 & & 0.02 & $\mathbf{0 . 0 0}$ & 0.03 & 0.04 & $\mathbf{0 . 0 0}$ & 0.03 \\
\hline Anoxic_A & 0.04 & 0.30 & 0.02 & 0.02 & & 0.01 & 0.03 & 0.03 & 0.02 & 0.02 \\
\hline Anoxic_B & 0.01 & 0.03 & 0.02 & $\mathbf{0 . 0 0}$ & 0.01 & & 0.04 & 0.04 & 0.02 & 0.03 \\
\hline Fermenter_A & $\mathbf{0 . 0 0}$ & 0.03 & 0.03 & 0.03 & 0.03 & 0.04 & & $\mathbf{0 . 0 0}$ & 0.01 & 0.02 \\
\hline Fermenter_B & 0.02 & 0.03 & 0.02 & 0.04 & 0.03 & 0.04 & $\mathbf{0 . 0 0}$ & & 0.01 & 0.03 \\
\hline PST_A & 0.05 & 0.01 & 0.01 & $\mathbf{0 . 0 0}$ & 0.02 & 0.02 & 0.01 & 0.01 & & 0.03 \\
\hline PST_B & 0.01 & 0.07 & 0.03 & 0.03 & 0.02 & 0.03 & 0.02 & 0.03 & 0.03 & \\
\hline
\end{tabular}

Similar observation was noted when comparing sampling zones in the order the wastewater flows through EBPR treatment process. Most of the Sørensen values from each pair were closely ranged $83-100 \%$ with the exception of the pairs anaerobic B and anoxic B, anaerobic A and aerobic A, and PST B and aerobic B, which were found

Table S3 Relative abundance (\%) of major phylogenetic groups within treatment zones of each wastewater treatment plant (EBPR)

\begin{tabular}{|c|c|c|c|c|c|c|c|c|c|c|}
\hline & \multicolumn{5}{|c|}{ Failed EBPR } & \multicolumn{5}{|c|}{ Successful EBPR } \\
\hline Name & $\begin{array}{c}\text { Aerobic } \\
\text { A }\end{array}$ & $\begin{array}{c}\text { Anoxic } \\
\text { A }\end{array}$ & $\begin{array}{c}\text { Anaerobic } \\
\text { A }\end{array}$ & $\begin{array}{c}\text { Fermenter } \\
\text { A }\end{array}$ & PSTA & $\begin{array}{c}\text { Aerobic } \\
\text { B }\end{array}$ & $\begin{array}{c}\text { Anoxic } \\
\text { B }\end{array}$ & $\begin{array}{c}\text { Anaerobic } \\
\text { B }\end{array}$ & $\begin{array}{c}\text { Fermenter } \\
\text { B }\end{array}$ & PSTB \\
\hline Firmicutes & 10.48 & 2.81 & 3.13 & 4.48 & 9.07 & 1.81 & 4.58 & 1.74 & 9.67 & 26.03 \\
\hline Proteobacteria & 75.81 & 79.48 & 75.42 & 57.04 & 74.09 & 85.39 & 80.29 & 85.36 & 30.7 & 61.7 \\
\hline Actinobacteria & 6.85 & 4.36 & 4.81 & 0.24 & 4.53 & 3.11 & 4.2 & 2.23 & 0.54 & 5.96 \\
\hline $\begin{array}{c}\text { Candidatus } \\
\text { Saccharibacteria }\end{array}$ & 0.81 & 0.07 & 0.00 & 0.00 & 4.15 & 0.11 & 0.00 & 0.17 & 0 & 1.15 \\
\hline Bacteroidetes & 4.44 & 5.27 & 6.03 & 34.91 & 4.27 & 2.49 & 4.05 & 1.74 & 55.84 & 4.24 \\
\hline Parcubacteria & 1.21 & 0.00 & 0.08 & 0.08 & 2.46 & 0.06 & 0.00 & 0.00 & 0.00 & 0.34 \\
\hline Chloroflexi & 0.2 & 4.43 & 5.34 & 0.00 & 1.04 & 1.13 & 2.06 & 2.23 & 0 & 0.23 \\
\hline Fusobacteria & 0.2 & 0.14 & 0.15 & 2.93 & 0.13 & 0.11 & 0.15 & 0.17 & 2.09 & 0.11 \\
\hline Lentisphaerae & 0.00 & 0.00 & 0.00 & 0.08 & 0.00 & 0.00 & 0.00 & 0.00 & 0.08 & 0.00 \\
\hline Verrucomicrobia & 0.00 & 1.05 & 1.53 & 0.16 & 0.13 & 0.91 & 0.31 & 1.9 & 0.08 & 0.00 \\
\hline Fibrobacteres & 0.00 & 0.00 & 0.00 & 0.00 & 0.00 & 0.00 & 0.08 & 0.08 & 1.01 & 0.00 \\
\hline Elusimicrobia & 0.00 & 0.00 & 0.00 & 0.00 & 0.13 & 0.00 & 0.08 & 0.00 & 0.00 & 0.00 \\
\hline Nitrospirae & 0.00 & 1.19 & 1.53 & 0.00 & 0.00 & 2.21 & 2.37 & 0.83 & 0.00 & 0.00 \\
\hline Planctomycetes & 0.00 & 0.91 & 1.15 & 0.00 & 0.00 & 2.21 & 1.53 & 3.31 & 0.00 & 0.11 \\
\hline Acidobacteria & 0.00 & 0.07 & 0.31 & 0.00 & 0.00 & 0.34 & 0.08 & 0.17 & 0.00 & 0.00 \\
\hline Spirochaetes & 0.00 & 0.00 & 0.31 & 0.00 & 0.00 & 0.00 & 0.15 & 0.00 & 0.00 & 0.00 \\
\hline Chlamydiae & 0.00 & 0.00 & 0.15 & 0.00 & 0.00 & 0.00 & 0.00 & 0.00 & 0.00 & 0.00 \\
\hline Armatimonadetes & 0.00 & 0.07 & 0.08 & 0.00 & 0.00 & 0.00 & 0.08 & 0.08 & 0.00 & 0.00 \\
\hline $\begin{array}{l}\text { Deinococcus- } \\
\text { Thermus }\end{array}$ & 0.00 & 0.07 & 0.00 & 0.00 & 0.00 & 0.06 & 0.00 & 0.00 & 0.00 & 0.11 \\
\hline Gemmatimonadetes & 0.00 & 0.07 & 0.00 & 0.00 & 0.00 & 0.00 & 0.00 & 0.00 & 0.00 & 0.00 \\
\hline
\end{tabular}


Table S4A. Abundances of all class in each sample of the Failed EBPR. The abundance is presented in terms of percentages of the total sequences in a sample.

\begin{tabular}{|c|c|c|c|c|c|}
\hline Name & Aerobic_A & Anaerobic_A & Anoxic_A & Fermenter_A & PST_A \\
\hline Betaproteobacteria & 44.35 & 40.41 & 45.01 & 13.45 & 44.24 \\
\hline Alphaproteobacteria & 12.11 & 5.42 & 5.34 & 0.57 & 11.79 \\
\hline Gammaproteobacteria & 14.37 & 4.74 & 6.05 & 30.07 & 12.48 \\
\hline Epsilonproteobacteria & 0.21 & 0.61 & 0.14 & 3.83 & 0.28 \\
\hline Deltaproteobacteria & 0.00 & 4.81 & 3.02 & 0.00 & 0.28 \\
\hline Actinobacteria & 6.98 & 4.81 & 4.36 & 0.24 & 4.72 \\
\hline Bacilli & 0.41 & 0.00 & 0.07 & 0.24 & 0.83 \\
\hline Erysipelotrichia & 0.41 & 0.00 & 0.28 & 0.00 & 0.28 \\
\hline Clostridia & 9.24 & 2.83 & 2.11 & 2.85 & 8.18 \\
\hline Negativicutes & 0.00 & 0.15 & 0.14 & 1.22 & 0.00 \\
\hline Flavobacteriia & 4.31 & 0.53 & 0.91 & 1.30 & 3.19 \\
\hline Sphingobacteriia & 0.21 & 0.92 & 0.98 & 0.00 & 0.00 \\
\hline Cytophagia & 0.00 & 0.31 & 0.07 & 0.00 & 0.00 \\
\hline Bacteroidetes_incertae_sedis & 0.00 & 0.08 & 0.00 & 0.00 & 0.00 \\
\hline Bacteroidia & 0.00 & 0.38 & 0.14 & 23.15 & 0.00 \\
\hline Fusobacteriia & 0.21 & 0.15 & 0.14 & 2.85 & 0.14 \\
\hline Anaerolineae & 0.21 & 4.51 & 4.15 & 0.00 & 0.55 \\
\hline Caldilineae & 0.00 & 0.38 & 0.07 & 0.00 & 0.42 \\
\hline Chloroflexia & 0.00 & 0.23 & 0.07 & 0.00 & 0.14 \\
\hline Nitrospira & 0.00 & 1.53 & 1.20 & 0.00 & 0.00 \\
\hline Planctomycetia & 0.00 & 1.15 & 0.91 & 0.00 & 0.00 \\
\hline Verrucomicrobiae & 0.00 & 0.38 & 0.07 & 0.00 & 0.14 \\
\hline Subdivision3 & 0.00 & 0.38 & 0.07 & 0.00 & 0.00 \\
\hline Opitutae & 0.00 & 0.61 & 0.77 & 0.16 & 0.00 \\
\hline Acidobacteria_Gp4 & 0.00 & 0.08 & 0.00 & 0.00 & 0.00 \\
\hline Holophagae & 0.00 & 0.15 & 0.00 & 0.00 & 0.00 \\
\hline Acidobacteria_Gp3 & 0.00 & 0.00 & 0.07 & 0.00 & 0.00 \\
\hline Spirochaetia & 0.00 & 0.31 & 0.00 & 0.00 & 0.00 \\
\hline Chlamydiia & 0.00 & 0.15 & 0.00 & 0.00 & 0.00 \\
\hline Fimbriimonadia & 0.00 & 0.00 & 0.07 & 0.00 & 0.00 \\
\hline Deinococci & 0.00 & 0.00 & 0.07 & 0.00 & 0.00 \\
\hline Gemmatimonadetes & 0.00 & 0.00 & 0.07 & 0.00 & 0.00 \\
\hline Lentisphaeria & 0.00 & 0.00 & 0.00 & 0.08 & 0.00 \\
\hline Chloroplast & 0.00 & 0.00 & 0.00 & 0.08 & 0.00 \\
\hline Elusimicrobia & 0.00 & 0.00 & 0.00 & 0.00 & 0.14 \\
\hline unclassified_Firmicutes & 0.62 & 0.15 & 0.21 & 0.16 & 0.55 \\
\hline unclassified_"Proteobacteria" & 6.37 & 19.48 & 19.97 & 9.21 & 10.12 \\
\hline unclassified_"Bacteroidetes" & 0.00 & 3.90 & 3.16 & 10.51 & 1.39 \\
\hline unclassified_"Acidobacteria" & 0.00 & 0.08 & 0.00 & 0.00 & 0.00 \\
\hline
\end{tabular}


Table S4A. Continued.

\begin{tabular}{|c|c|c|c|c|c|}
\hline unclassified_"Chloroflexi” & 0.00 & 0.23 & 0.14 & 0.00 & 0.00 \\
\hline unclassified_"Verrucomicrobia" & 0.00 & 0.15 & 0.14 & 0.00 & 0.00 \\
\hline unclassified_"Actinobacteria" & 0.00 & 0.00 & 0.00 & 0.00 & 0.14 \\
\hline
\end{tabular}

Table S4B. Abundances of all class in each sample of the Successful EBPR. The abundance is presented in terms of percentages of the total sequences in a sample.

\begin{tabular}{|c|c|c|c|c|c|}
\hline Name & Aerobic_B & Anaerobic_B & Anoxic_B & Fermenter_B & PST_B \\
\hline Planctomycetia & 2.21 & 3.24 & 1.53 & 0.00 & 0.12 \\
\hline Phycisphaerae & 0.00 & 0.08 & 0.00 & 0.00 & 0.00 \\
\hline Betaproteobacteria & 45.74 & 61.41 & 37.23 & 8.19 & 35.39 \\
\hline Gammaproteobacteria & 14.98 & 4.56 & 19.27 & 16.23 & 14.78 \\
\hline Alphaproteobacteria & 6.24 & 1.16 & 4.43 & 0.31 & 5.59 \\
\hline Deltaproteobacteria & 1.65 & 2.57 & 1.76 & 0.08 & 0.00 \\
\hline Epsilonproteobacteria & 0.00 & 0.08 & 0.92 & 1.16 & 0.00 \\
\hline Anaerolineae & 0.91 & 1.83 & 1.68 & 0.00 & 0.00 \\
\hline Caldilineae & 0.11 & 0.33 & 0.31 & 0.00 & 0.23 \\
\hline Chloroflexia & 0.00 & 0.00 & 0.08 & 0.00 & 0.00 \\
\hline Fusobacteriia & 0.11 & 0.17 & 0.15 & 2.09 & 0.12 \\
\hline Flavobacteriia & 0.34 & 0.17 & 0.38 & 0.77 & 3.49 \\
\hline Sphingobacteriia & 0.34 & 0.41 & 0.23 & 0.00 & 0.58 \\
\hline Bacteroidia & 0.11 & 0.17 & 1.30 & 38.49 & 0.00 \\
\hline Bacteroidetes_incertae_sedis & 0.11 & 0.00 & 0.08 & 0.08 & 0.00 \\
\hline Cytophagia & 0.00 & 0.00 & 0.23 & 0.00 & 0.00 \\
\hline Nitrospira & 2.21 & 0.83 & 2.37 & 0.00 & 0.00 \\
\hline Actinobacteria & 3.06 & 2.16 & 4.20 & 0.54 & 5.94 \\
\hline Subdivision3 & 0.23 & 0.25 & 0.08 & 0.00 & 0.00 \\
\hline Verrucomicrobiae & 0.28 & 0.25 & 0.15 & 0.00 & 0.00 \\
\hline Opitutae & 0.34 & 1.24 & 0.08 & 0.08 & 0.00 \\
\hline Acidobacteria_Gp3 & 0.11 & 0.00 & 0.08 & 0.00 & 0.00 \\
\hline Acidobacteria_Gp6 & 0.06 & 0.00 & 0.00 & 0.00 & 0.00 \\
\hline Acidobacteria_Gp4 & 0.00 & 0.17 & 0.00 & 0.00 & 0.00 \\
\hline Clostridia & 1.53 & 1.66 & 3.44 & 7.81 & 22.12 \\
\hline Bacilli & 0.11 & 0.00 & 0.15 & 0.00 & 1.51 \\
\hline Erysipelotrichia & 0.06 & 0.08 & 0.08 & 0.15 & 1.16 \\
\hline Negativicutes & 0.00 & 0.00 & 0.31 & 1.08 & 0.00 \\
\hline Deinococci & 0.06 & 0.00 & 0.00 & 0.00 & 0.12 \\
\hline Fibrobacteria & 0.00 & 0.08 & 0.08 & 1.00 & 0.00 \\
\hline Armatimonadia & 0.00 & 0.08 & 0.00 & 0.00 & 0.00 \\
\hline Elusimicrobia & 0.00 & 0.00 & 0.08 & 0.00 & 0.00 \\
\hline Spirochaetia & 0.00 & 0.00 & 0.15 & 0.00 & 0.00 \\
\hline
\end{tabular}


Table S4B. Continued.

\begin{tabular}{|c|c|c|c|c|c|}
\hline Lentisphaeria & 0.00 & 0.00 & 0.00 & 0.08 & 0.00 \\
\hline unclassified_"Proteobacteria" & 16.91 & 15.68 & 16.67 & 4.71 & 6.87 \\
\hline unclassified_"Bacteroidetes" & 1.65 & 1.00 & 1.83 & 16.54 & 0.23 \\
\hline unclassified_"Chloroflexi" & 0.11 & 0.08 & 0.00 & 0.00 & 0.00 \\
\hline unclassified_"Acidobacteria" & 0.17 & 0.00 & 0.00 & 0.00 & 0.00 \\
\hline unclassified_"Verrucomicrobia" & 0.06 & 0.17 & 0.00 & 0.00 & 0.00 \\
\hline unclassified_Firmicutes & 0.11 & 0.00 & 0.61 & 0.62 & 1.63 \\
\hline unclassified_"Actinobacteria" & 0.06 & 0.08 & 0.00 & 0.00 & 0.12 \\
\hline unclassified_"Armatimonadetes" & 0.00 & 0.00 & 0.08 & 0.00 & 0.00 \\
\hline
\end{tabular}

to be below $80 \%$, highlighting a slightly similar level of diversity between pairs (Fig. 3). Similarly, the Jaccard values also showed results ranging $65-100 \%$. Another statistical analysis using the UniFrac metric analysis was performed to further analyse the similarity between samples by taking into consideration not only the presence or abundance of OTUs, but also the bacterial phylogeny [30]. Similarly to the Jaccard and Sørensen index, UniFrac metrics revealed a high significant shift $(\mathrm{p}>0.05)$ among the microbial community of sampling zones with the exception of anaerobic_A/aerobic_A and PST_B / aerobic_B, highlighting no or slightly similar levels of diversity (Table S2). This finding disagrees with the results by Zhang et al. [31], who reported a similar level of diversity between the bacterial structure and activity of 14 EBPR. Lawson et al. [32] also stated that functional similarity in several EBPRs is mostly translated by the number of OTUs having similar genus-level affiliation. A significant shift of the dominant microbial community highlighting high dissimilarity between anaerobic-anoxic has also been reported by Lv et al. [21] when comparing the bacterial community in EBPRs.

\section{Bacterial Community Structure in Both Wastewater Treatment Plants}

In order to comparatively analyse the bacterial community from the two different wastewater treatment plants, the RDP classifier was used to assign the readings to specific phylogenetic bacterial taxa. The results showed 19 bacterial phyla with the exception of unclassified bacterial readings from both failed and successful EBPRs. Both EBPRs had 17 shared phyla and two unique phyla for failed EBPRs (Chlamydiae and Gemmatimonadetes) and two unique phyla for successful EBPRs (Microgenomates and Fibrobacteres) (Table S3, Fig. 4). The observed unique phyla have similarly been reported elsewhere in low proportions in activated sludge $[5,8,32]$. In terms of their abundance in both plants separately, five phyla - Proteobacteria (72.37\%), Bacteriodetes, Firmicutes, Actinobacteria, and Chloroflexi - occupied approximately $95.71 \%$ of all classified bacteria from the failed EBPR, while in the successful EBPR Proteobacteria, Bacteriodetes, Firmicutes, Actinobacteria, and Planctomycetes were the most abundant with approximately $95.77 \%$ of the entire classified bacterial population (Fig. 3). This study is in agreement with Tian et al. [32], who also reported the predominance of Proteobacteria, Bacteriodetes, Firmicutes, Actinobacteria, and Planctomycetes in wastewater treatment plants containing approximately 51 phyla and 900 genera. Bond et al. [8], in their study investigating the bacterial community structures of phosphate-removing and non-phosphate-removing activated sludges from sequencing batch reactors, also revealed that Proteobacteria, Planctomycete, Flexibacter, Cytophaga, and Bacteroides were the dominant microbial groups.

The presence of Proteobacteria in EBPR as the most predominant phylum followed by Bacteriodetes as the second most predominant phylum has also been reported by Albertsen et al. [4]. This has been confirmed by Ye and Zhang [20], who reported Proteobacteria as the predominant bacterial phylum in several activated sludge samples. Furthermore, the predominance of Bacteriodetes over Proteobacteria in EBPR or activated sludge has not been intensively reported [32-33].

It was also noted that the bacterial community from both plants become more diverse as the sequences were classified into lower taxonomic levels (Table S47). Despite the high diversity, many readings could not be classified into a lower taxonomic level. This could be explained as Jin et al. [34] reported that up to 80$90 \%$ of microorganisms in activated sludge cannot be cultured and that the sludge harbours a community of largely uninvestigated novel species. In the present study, a total of approximately $49.64 \%$ of readings and $58.52 \%$ of readings from failed and successful EBPR plants, respectively, could not be classified up to a genus level (Table S3-S7). In addition, in both plants $21.47 \%$ of readings $(1,430)$ from failed EBPR and $17.18 \%$ of readings $(1,337)$ from successful EBPR could also not be classified at the phylum level. This result was in disagreement with the microbial pattern as reported by Feng et al. [35]. 
Table S5A. Abundances of all order in each sample of the Failed EBPR. The abundance is presented in terms of percentages of the total sequences in a sample

\begin{tabular}{|c|c|c|c|c|c|}
\hline Name & Aerobic_A & Anaerobic_A & Anoxic_A & Fermenter_A & PST_A \\
\hline Burkholderiales & 43.05 & 8.50 & 8.85 & 9.67 & 42.50 \\
\hline Rhodocyclales & 0.88 & 22.27 & 25.62 & 1.43 & 1.26 \\
\hline Neisseriales & 0.00 & 0.30 & 0.00 & 0.51 & 0.16 \\
\hline Ferrovales & 0.00 & 0.51 & 0.00 & 0.00 & 0.00 \\
\hline Nitrosomonadales & 0.00 & 0.51 & 0.74 & 0.00 & 0.32 \\
\hline Rhizobiales & 1.55 & 1.92 & 2.12 & 0.10 & 2.37 \\
\hline Caulobacterales & 5.96 & 0.10 & 0.00 & 0.00 & 4.42 \\
\hline Sphingomonadales & 0.66 & 0.10 & 0.46 & 0.00 & 1.58 \\
\hline Rhodobacterales & 0.88 & 0.91 & 1.38 & 0.61 & 1.26 \\
\hline Rhodospirillales & 0.22 & 0.40 & 0.46 & 0.00 & 0.63 \\
\hline Enterobacteriales & 3.09 & 0.20 & 0.28 & 1.83 & 0.95 \\
\hline Pseudomonadales & 5.30 & 1.62 & 1.94 & 13.24 & 5.21 \\
\hline Oceanospirillales & 0.22 & 0.00 & 0.00 & 0.00 & 1.11 \\
\hline Alteromonadales & 0.66 & 0.00 & 0.00 & 0.00 & 0.79 \\
\hline Xanthomonadales & 0.44 & 0.51 & 1.47 & 0.00 & 0.16 \\
\hline Aeromonadales & 0.22 & 0.71 & 0.92 & 7.84 & 0.16 \\
\hline Chromatiales & 0.00 & 0.00 & 0.00 & 0.00 & 0.16 \\
\hline Thiotrichales & 0.00 & 0.00 & 0.00 & 0.00 & 0.16 \\
\hline Campylobacterales & 0.22 & 0.71 & 0.18 & 4.79 & 0.32 \\
\hline Myxococcales & 0.00 & 5.67 & 3.32 & 0.00 & 0.00 \\
\hline Bdellovibrionales & 0.00 & 0.00 & 0.00 & 0.00 & 0.32 \\
\hline Actinomycetales & 6.18 & 3.54 & 3.41 & 0.20 & 4.74 \\
\hline Bifidobacteriales & 0.00 & 0.20 & 0.00 & 0.10 & 0.00 \\
\hline Coriobacteriales & 0.22 & 0.00 & 0.00 & 0.00 & 0.00 \\
\hline Solirubrobacterales & 0.44 & 0.00 & 0.00 & 0.00 & 0.00 \\
\hline Acidimicrobiales & 0.22 & 1.42 & 1.11 & 0.00 & 0.00 \\
\hline Lactobacillales & 0.22 & 0.00 & 0.09 & 0.31 & 0.32 \\
\hline Bacillales & 0.22 & 0.00 & 0.00 & 0.00 & 0.47 \\
\hline Erysipelotrichales & 0.44 & 0.00 & 0.37 & 0.00 & 0.32 \\
\hline Clostridiales & 9.93 & 3.74 & 2.76 & 3.56 & 9.32 \\
\hline Selenomonadales & 0.00 & 0.20 & 0.18 & 1.53 & 0.00 \\
\hline Flavobacteriales & 4.64 & 0.71 & 1.20 & 1.63 & 3.63 \\
\hline Sphingobacteriales & 0.22 & 1.21 & 1.29 & 0.00 & 0.00 \\
\hline Cytophagales & 0.00 & 0.40 & 0.09 & 0.00 & 0.00 \\
\hline Bacteroidales & 0.00 & 0.51 & 0.18 & 28.92 & 0.00 \\
\hline Fusobacteriales & 0.22 & 0.20 & 0.18 & 3.56 & 0.16 \\
\hline Anaerolineales & 0.22 & 5.97 & 5.44 & 0.00 & 0.63 \\
\hline Caldilineales & 0.00 & 0.51 & 0.09 & 0.00 & 0.47 \\
\hline Chloroflexales & 0.00 & 0.30 & 0.09 & 0.00 & 0.00 \\
\hline
\end{tabular}


Table S5A. Continued.

\begin{tabular}{|c|c|c|c|c|c|}
\hline Herpetosiphonales & 0.00 & 0.00 & 0.00 & 0.00 & 0.16 \\
\hline Nitrospirales & 0.00 & 2.02 & 1.57 & 0.00 & 0.00 \\
\hline Planctomycetales & 0.00 & 1.52 & 1.20 & 0.00 & 0.00 \\
\hline Verrucomicrobiales & 0.00 & 0.51 & 0.09 & 0.00 & 0.16 \\
\hline Opitutales & 0.00 & 0.81 & 0.92 & 0.20 & 0.00 \\
\hline Holophagales & 0.00 & 0.20 & 0.00 & 0.00 & 0.00 \\
\hline Spirochaetales & 0.00 & 0.40 & 0.00 & 0.00 & 0.00 \\
\hline Chlamydiales & 0.00 & 0.20 & 0.00 & 0.00 & 0.00 \\
\hline Fimbriimonadales & 0.00 & 0.00 & 0.09 & 0.00 & 0.00 \\
\hline Gemmatimonadales & 0.00 & 0.00 & 0.09 & 0.00 & 0.00 \\
\hline Victivallales & 0.00 & 0.00 & 0.00 & 0.10 & 0.00 \\
\hline Elusimicrobiales & 0.00 & 0.00 & 0.00 & 0.00 & 0.16 \\
\hline unclassified_Alphaproteobacteria & 3.75 & 3.74 & 2.58 & 0.00 & 3.16 \\
\hline unclassified_Gammaproteobacteria & 5.52 & 3.24 & 3.32 & 14.66 & 5.53 \\
\hline unclassified_Betaproteobacteria & 3.75 & 21.46 & 23.78 & 5.19 & 6.16 \\
\hline unclassified_Deltaproteobacteria & 0.00 & 0.71 & 0.65 & 0.00 & 0.00 \\
\hline unclassified_Epsilonproteobacteria & 0.00 & 0.10 & 0.00 & 0.00 & 0.00 \\
\hline unclassified_Rubrobacteridae & 0.00 & 0.10 & 0.00 & 0.00 & 0.00 \\
\hline unclassified_Actinobacteria & 0.44 & 1.11 & 1.20 & 0.00 & 0.63 \\
\hline unclassified_Bacilli & 0.00 & 0.00 & 0.00 & 0.00 & 0.16 \\
\hline unclassified_Opitutae & 0.00 & 0.00 & 0.09 & 0.00 & 0.00 \\
\hline unclassified_Acidobacteria_Gp3 & 0.00 & 0.00 & 0.09 & 0.00 & 0.00 \\
\hline unclassified_Deinococci & 0.00 & 0.00 & 0.09 & 0.00 & 0.00 \\
\hline
\end{tabular}

Table S5B. Abundances of all order in each sample of the Successful EBPR. The abundance is presented in terms of percentages of the total sequences in a sample.

\begin{tabular}{|c|c|c|c|c|c|}
\hline Name & Aerobic_B & Anaerobic_B & Anoxic_B & Fermenter_B & PST_B \\
\hline Planctomycetales & 2.75 & 3.82 & 1.90 & 0.00 & 0.13 \\
\hline Phycisphaerales & 0.00 & 0.10 & 0.00 & 0.00 & 0.00 \\
\hline Rhodocyclales & 24.45 & 34.17 & 15.92 & 0.50 & 0.77 \\
\hline Burkholderiales & 8.32 & 11.56 & 8.72 & 6.73 & 32.57 \\
\hline Nitrosomonadales & 0.49 & 1.01 & 0.57 & 0.00 & 0.00 \\
\hline Ferrovales & 0.14 & 0.20 & 0.00 & 0.00 & 0.00 \\
\hline Neisseriales & 0.00 & 0.00 & 0.09 & 0.40 & 0.00 \\
\hline Enterobacteriales & 7.68 & 0.00 & 9.67 & 0.89 & 0.77 \\
\hline Pseudomonadales & 0.85 & 0.30 & 1.23 & 9.80 & 1.92 \\
\hline Xanthomonadales & 0.21 & 0.10 & 0.47 & 0.20 & 0.38 \\
\hline Aeromonadales & 0.28 & 0.00 & 0.47 & 4.36 & 1.02 \\
\hline Oceanospirillales & 0.00 & 0.00 & 0.00 & 0.00 & 1.02 \\
\hline Alteromonadales & 0.00 & 0.00 & 0.00 & 0.00 & 1.66 \\
\hline
\end{tabular}


Table S5B. Continued.

\begin{tabular}{|c|c|c|c|c|c|}
\hline Rhizobiales & 1.55 & 0.30 & 1.61 & 0.10 & 1.02 \\
\hline Rhodobacterales & 2.18 & 0.10 & 0.85 & 0.20 & 1.02 \\
\hline Sphingomonadales & 0.63 & 0.50 & 0.28 & 0.00 & 0.51 \\
\hline Rhodospirillales & 0.21 & 0.00 & 0.09 & 0.00 & 0.13 \\
\hline Caulobacterales & 0.07 & 0.00 & 0.09 & 0.00 & 2.04 \\
\hline Myxococcales & 1.69 & 2.41 & 1.52 & 0.00 & 0.00 \\
\hline Bdellovibrionales & 0.14 & 0.30 & 0.19 & 0.00 & 0.00 \\
\hline Desulfovibrionales & 0.00 & 0.10 & 0.00 & 0.10 & 0.00 \\
\hline Campylobacterales & 0.00 & 0.10 & 1.14 & 1.49 & 0.00 \\
\hline Anaerolineales & 1.13 & 2.21 & 2.09 & 0.00 & 0.00 \\
\hline Caldilineales & 0.14 & 0.40 & 0.38 & 0.00 & 0.26 \\
\hline Chloroflexales & 0.00 & 0.00 & 0.09 & 0.00 & 0.00 \\
\hline Fusobacteriales & 0.14 & 0.20 & 0.19 & 2.67 & 0.13 \\
\hline Flavobacteriales & 0.42 & 0.20 & 0.47 & 0.99 & 3.83 \\
\hline Sphingobacteriales & 0.42 & 0.50 & 0.28 & 0.00 & 0.64 \\
\hline Bacteroidales & 0.14 & 0.20 & 1.61 & 49.31 & 0.00 \\
\hline Cytophagales & 0.00 & 0.00 & 0.28 & 0.00 & 0.00 \\
\hline Nitrospirales & 2.75 & 1.01 & 2.94 & 0.00 & 0.00 \\
\hline Acidimicrobiales & 0.78 & 0.20 & 0.57 & 0.00 & 0.26 \\
\hline Actinomycetales & 2.47 & 1.71 & 3.89 & 0.59 & 6.00 \\
\hline Bifidobacteriales & 0.07 & 0.00 & 0.09 & 0.00 & 0.00 \\
\hline Solirubrobacterales & 0.14 & 0.00 & 0.19 & 0.00 & 0.00 \\
\hline Coriobacteriales & 0.00 & 0.00 & 0.00 & 0.10 & 0.00 \\
\hline Verrucomicrobiales & 0.35 & 0.30 & 0.19 & 0.00 & 0.00 \\
\hline Opitutales & 0.42 & 1.51 & 0.09 & 0.00 & 0.00 \\
\hline Clostridiales & 1.90 & 2.01 & 4.27 & 9.90 & 24.27 \\
\hline Lactobacillales & 0.14 & 0.00 & 0.09 & 0.00 & 0.26 \\
\hline Bacillales & 0.00 & 0.00 & 0.09 & 0.00 & 1.40 \\
\hline Erysipelotrichales & 0.07 & 0.10 & 0.09 & 0.20 & 1.28 \\
\hline Selenomonadales & 0.00 & 0.00 & 0.38 & 1.39 & 0.00 \\
\hline Deinococcales & 0.00 & 0.00 & 0.00 & 0.00 & 0.13 \\
\hline Fibrobacterales & 0.00 & 0.10 & 0.09 & 1.29 & 0.00 \\
\hline Armatimonadales & 0.00 & 0.10 & 0.00 & 0.00 & 0.00 \\
\hline Elusimicrobiales & 0.00 & 0.00 & 0.09 & 0.00 & 0.00 \\
\hline Spirochaetales & 0.00 & 0.00 & 0.19 & 0.00 & 0.00 \\
\hline Victivallales & 0.00 & 0.00 & 0.00 & 0.10 & 0.00 \\
\hline unclassified_Planctomycetia & 0.00 & 0.10 & 0.00 & 0.00 & 0.00 \\
\hline unclassified_Betaproteobacteria & 23.40 & 27.44 & 20.85 & 2.87 & 5.49 \\
\hline unclassified_Gammaproteobacteria & 9.58 & 5.13 & 12.04 & 5.54 & 9.45 \\
\hline unclassified_Alphaproteobacteria & 3.10 & 0.50 & 2.56 & 0.10 & 1.40 \\
\hline
\end{tabular}


Table S5B. Continued.

\begin{tabular}{|c|c|c|c|c|c|}
\hline unclassified_Deltaproteobacteria & 0.21 & 0.30 & 0.47 & 0.00 & 0.00 \\
\hline unclassified_Actinobacteria & 0.35 & 0.70 & 0.47 & 0.00 & 0.26 \\
\hline unclassified_Opitutae & 0.00 & 0.00 & 0.00 & 0.10 & 0.00 \\
\hline unclassified_Acidobacteria_Gp3 & 0.14 & 0.00 & 0.09 & 0.00 & 0.00 \\
\hline unclassified_Deinococci & 0.07 & 0.00 & 0.00 & 0.00 & 0.00 \\
\hline unclassified_Clostridia & 0.00 & 0.00 & 0.00 & 0.10 & 0.00 \\
\hline
\end{tabular}

Table S6A. Abundances of all family in each sample of the Failed EBPR. The abundance is presented in terms of percentages of the total sequences in a sample.

\begin{tabular}{|c|c|c|c|c|c|}
\hline Name & Aerobic_A & Anaerobic_A & Anoxic_A & Fermenter_A & PST_A \\
\hline Comamonadaceae & 38.01 & 4.95 & 4.73 & 11.29 & 28.09 \\
\hline Burkholderiaceae & 4.34 & 0.00 & 0.27 & 0.00 & 12.55 \\
\hline Oxalobacteraceae & 2.30 & 0.00 & 0.00 & 0.00 & 3.18 \\
\hline Burkholderiales_incertae_sedis & 1.28 & 2.62 & 2.97 & 0.00 & 1.31 \\
\hline Alcaligenaceae & 0.00 & 0.00 & 0.41 & 0.00 & 0.19 \\
\hline Rhodocyclaceae & 1.02 & 32.02 & 37.57 & 1.78 & 1.50 \\
\hline Neisseriaceae & 0.00 & 0.44 & 0.00 & 0.63 & 0.19 \\
\hline Ferrovaceae & 0.00 & 0.73 & 0.00 & 0.00 & 0.00 \\
\hline Nitrosomonadaceae & 0.00 & 0.73 & 1.08 & 0.00 & 0.37 \\
\hline Phyllobacteriaceae & 0.26 & 0.29 & 0.27 & 0.00 & 0.75 \\
\hline Methylobacteriaceae & 0.51 & 0.00 & 0.00 & 0.00 & 0.19 \\
\hline Hyphomicrobiaceae & 0.26 & 0.00 & 0.27 & 0.13 & 0.19 \\
\hline Bradyrhizobiaceae & 0.00 & 0.87 & 0.95 & 0.00 & 0.19 \\
\hline Rhizobiaceae & 0.00 & 0.15 & 0.00 & 0.00 & 0.00 \\
\hline Methylocystaceae & 0.00 & 0.00 & 0.14 & 0.00 & 0.00 \\
\hline Beijerinckiaceae & 0.00 & 0.00 & 0.00 & 0.00 & 0.19 \\
\hline Caulobacteraceae & 6.89 & 0.15 & 0.00 & 0.00 & 5.24 \\
\hline Sphingomonadaceae & 0.77 & 0.15 & 0.41 & 0.00 & 1.69 \\
\hline Erythrobacteraceae & 0.00 & 0.00 & 0.00 & 0.00 & 0.19 \\
\hline Rhodobacteraceae & 1.02 & 1.31 & 2.03 & 0.76 & 1.50 \\
\hline Acetobacteraceae & 0.26 & 0.29 & 0.27 & 0.00 & 0.00 \\
\hline Rhodospirillaceae & 0.00 & 0.29 & 0.41 & 0.00 & 0.75 \\
\hline Enterobacteriaceae & 3.57 & 0.29 & 0.41 & 2.28 & 1.12 \\
\hline Pseudomonadaceae & 5.61 & 0.87 & 1.76 & 0.89 & 4.12 \\
\hline Moraxellaceae & 0.51 & 1.31 & 0.95 & 15.23 & 1.87 \\
\hline Halomonadaceae & 0.26 & 0.00 & 0.00 & 0.00 & 1.31 \\
\hline Shewanellaceae & 0.77 & 0.00 & 0.00 & 0.00 & 0.75 \\
\hline Xanthomonadaceae & 0.51 & 0.73 & 2.16 & 0.00 & 0.19 \\
\hline Aeromonadaceae & 0.26 & 1.02 & 1.35 & 9.77 & 0.19 \\
\hline Chromatiaceae & 0.00 & 0.00 & 0.00 & 0.00 & 0.19 \\
\hline
\end{tabular}


Table S6A. Continued.

\begin{tabular}{|c|c|c|c|c|c|}
\hline Thiotrichaceae & 0.00 & 0.00 & 0.00 & 0.00 & 0.19 \\
\hline Campylobacteraceae & 0.26 & 1.02 & 0.14 & 5.84 & 0.37 \\
\hline Polyangiaceae & 0.00 & 1.89 & 0.54 & 0.00 & 0.00 \\
\hline Nannocystaceae & 0.00 & 3.78 & 2.84 & 0.00 & 0.00 \\
\hline Haliangiaceae & 0.00 & 0.15 & 0.00 & 0.00 & 0.00 \\
\hline Kofleriaceae & 0.00 & 0.15 & 0.14 & 0.00 & 0.00 \\
\hline Bacteriovoracaceae & 0.00 & 0.00 & 0.00 & 0.00 & 0.19 \\
\hline Bdellovibrionaceae & 0.00 & 0.00 & 0.00 & 0.00 & 0.19 \\
\hline Geodermatophilaceae & 0.26 & 0.00 & 0.00 & 0.00 & 0.94 \\
\hline Nocardiaceae & 0.26 & 0.15 & 0.00 & 0.00 & 0.00 \\
\hline Mycobacteriaceae & 0.00 & 0.00 & 0.54 & 0.00 & 0.00 \\
\hline Corynebacteriaceae & 0.00 & 0.00 & 0.00 & 0.00 & 0.19 \\
\hline Microbacteriaceae & 1.02 & 0.15 & 0.00 & 0.00 & 0.75 \\
\hline Intrasporangiaceae & 0.00 & 1.16 & 0.54 & 0.00 & 0.00 \\
\hline Dermacoccaceae & 0.00 & 0.15 & 0.00 & 0.00 & 0.00 \\
\hline Micrococcaceae & 0.00 & 0.00 & 0.00 & 0.00 & 0.19 \\
\hline Nocardioidaceae & 0.26 & 0.87 & 0.54 & 0.00 & 0.94 \\
\hline Propionibacteriaceae & 0.00 & 0.00 & 0.14 & 0.13 & 0.37 \\
\hline Actinomycetaceae & 0.26 & 0.00 & 0.00 & 0.00 & 0.19 \\
\hline Bifidobacteriaceae & 0.00 & 0.29 & 0.00 & 0.13 & 0.00 \\
\hline Coriobacteriaceae & 0.26 & 0.00 & 0.00 & 0.00 & 0.00 \\
\hline Iamiaceae & 0.00 & 0.87 & 0.00 & 0.00 & 0.00 \\
\hline Acidimicrobiaceae & 0.00 & 0.29 & 0.41 & 0.00 & 0.00 \\
\hline Streptococcaceae & 0.26 & 0.00 & 0.14 & 0.38 & 0.19 \\
\hline Bacillaceae 1 & 0.00 & 0.00 & 0.00 & 0.00 & 0.56 \\
\hline Erysipelotrichaceae & 0.51 & 0.00 & 0.54 & 0.00 & 0.37 \\
\hline Peptostreptococcaceae & 4.08 & 1.46 & 1.35 & 0.63 & 4.12 \\
\hline Clostridiaceae 1 & 5.61 & 1.46 & 1.35 & 0.89 & 3.00 \\
\hline Ruminococcaceae & 0.77 & 0.29 & 0.14 & 1.65 & 1.50 \\
\hline Lachnospiraceae & 0.00 & 0.87 & 0.41 & 0.63 & 0.94 \\
\hline Acidaminococcaceae & 0.00 & 0.15 & 0.00 & 0.76 & 0.00 \\
\hline Veillonellaceae & 0.00 & 0.00 & 0.14 & 0.76 & 0.00 \\
\hline Flavobacteriaceae & 5.36 & 1.02 & 1.49 & 1.90 & 4.12 \\
\hline Chitinophagaceae & 0.26 & 1.46 & 1.08 & 0.00 & 0.00 \\
\hline Saprospiraceae & 0.00 & 0.29 & 0.41 & 0.00 & 0.00 \\
\hline Sphingobacteriaceae & 0.00 & 0.00 & 0.27 & 0.00 & 0.00 \\
\hline Cytophagaceae & 0.00 & 0.58 & 0.00 & 0.00 & 0.00 \\
\hline Prevotellaceae & 0.00 & 0.58 & 0.14 & 21.95 & 0.00 \\
\hline Bacteroidaceae & 0.00 & 0.00 & 0.00 & 0.51 & 0.00 \\
\hline Porphyromonadaceae & 0.00 & 0.00 & 0.00 & 0.25 & 0.00 \\
\hline Leptotrichiaceae & 0.00 & 0.15 & 0.14 & 1.02 & 0.00 \\
\hline
\end{tabular}


Table S6A. Continued.

\begin{tabular}{|c|c|c|c|c|c|}
\hline Fusobacteriaceae & 0.00 & 0.00 & 0.14 & 1.02 & 0.19 \\
\hline Anaerolineaceae & 0.26 & 8.59 & 7.97 & 0.00 & 0.75 \\
\hline Caldilineaceae & 0.00 & 0.73 & 0.14 & 0.00 & 0.56 \\
\hline Chloroflexaceae & 0.00 & 0.29 & 0.00 & 0.00 & 0.00 \\
\hline Herpetosiphonaceae & 0.00 & 0.00 & 0.00 & 0.00 & 0.19 \\
\hline Nitrospiraceae & 0.00 & 2.91 & 2.30 & 0.00 & 0.00 \\
\hline Planctomycetaceae & 0.00 & 2.18 & 1.76 & 0.00 & 0.00 \\
\hline Verrucomicrobiaceae & 0.00 & 0.73 & 0.14 & 0.00 & 0.19 \\
\hline Opitutaceae & 0.00 & 1.16 & 1.35 & 0.25 & 0.00 \\
\hline Holophagaceae & 0.00 & 0.29 & 0.00 & 0.00 & 0.00 \\
\hline Leptospiraceae & 0.00 & 0.58 & 0.00 & 0.00 & 0.00 \\
\hline Fimbriimonadaceae & 0.00 & 0.00 & 0.14 & 0.00 & 0.00 \\
\hline Gemmatimonadaceae & 0.00 & 0.00 & 0.14 & 0.00 & 0.00 \\
\hline Victivallaceae & 0.00 & 0.00 & 0.00 & 0.13 & 0.00 \\
\hline Chloroplast & 0.00 & 0.00 & 0.00 & 0.13 & 0.00 \\
\hline Elusimicrobiaceae & 0.00 & 0.00 & 0.00 & 0.00 & 0.19 \\
\hline unclassified_Burkholderiales & 3.83 & 4.66 & 4.59 & 0.76 & 5.06 \\
\hline unclassified_Rhizobiales & 0.77 & 1.46 & 1.49 & 0.00 & 1.31 \\
\hline unclassified_Sphingomonadales & 0.00 & 0.00 & 0.27 & 0.00 & 0.00 \\
\hline unclassified_Pseudomonadales & 0.00 & 0.15 & 0.14 & 0.38 & 0.19 \\
\hline unclassified_Alteromonadales & 0.00 & 0.00 & 0.00 & 0.00 & 0.19 \\
\hline unclassified_Campylobacterales & 0.00 & 0.00 & 0.14 & 0.13 & 0.00 \\
\hline unclassified_Sorangiineae & 0.00 & 0.29 & 0.14 & 0.00 & 0.00 \\
\hline unclassified_Nannocystineae & 0.00 & 0.15 & 0.14 & 0.00 & 0.00 \\
\hline unclassified_Myxococcales & 0.00 & 1.75 & 1.08 & 0.00 & 0.00 \\
\hline unclassified_Corynebacterineae & 1.28 & 0.29 & 0.68 & 0.00 & 0.19 \\
\hline unclassified_Micrococcineae & 0.00 & 0.00 & 0.27 & 0.13 & 0.37 \\
\hline unclassified_Actinomycetales & 3.83 & 2.33 & 2.30 & 0.00 & 1.50 \\
\hline unclassified_Solirubrobacterales & 0.51 & 0.00 & 0.00 & 0.00 & 0.00 \\
\hline unclassified_"Acidimicrobineae" & 0.26 & 0.87 & 1.22 & 0.00 & 0.00 \\
\hline unclassified_Bacillales & 0.26 & 0.00 & 0.00 & 0.00 & 0.00 \\
\hline unclassified_Lactobacillales & 0.00 & 0.00 & 0.00 & 0.00 & 0.19 \\
\hline unclassified_Clostridiales & 1.02 & 1.31 & 0.81 & 0.63 & 1.50 \\
\hline unclassified_Selenomonadales & 0.00 & 0.15 & 0.14 & 0.38 & 0.00 \\
\hline unclassified_"Flavobacteriales" & 0.00 & 0.00 & 0.27 & 0.13 & 0.19 \\
\hline unclassified_"Sphingobacteriales" & 0.00 & 0.00 & 0.14 & 0.00 & 0.00 \\
\hline unclassified_Cytophagales & 0.00 & 0.00 & 0.14 & 0.00 & 0.00 \\
\hline unclassified_"Bacteroidales" & 0.00 & 0.15 & 0.14 & 13.32 & 0.00 \\
\hline unclassified_"Fusobacteriales" & 0.26 & 0.15 & 0.00 & 2.41 & 0.00 \\
\hline unclassified_Chloroflexineae & 0.00 & 0.15 & 0.14 & 0.00 & 0.00 \\
\hline unclassified_Chlamydiales & 0.00 & 0.29 & 0.00 & 0.00 & 0.00 \\
\hline
\end{tabular}


Table S6B. Abundances of all family in each sample of the Successful EBPR. The abundance is presented in terms of percentages of the total sequences in a sample.

\begin{tabular}{|c|c|c|c|c|c|}
\hline Name & Aerobic_B & Anaerobic_B & Anoxic_B & Fermenter_B & PST_B \\
\hline Planctomycetaceae & 4.35 & 5.80 & 2.99 & 0.00 & 0.15 \\
\hline Phycisphaeraceae & 0.00 & 0.15 & 0.00 & 0.00 & 0.00 \\
\hline Rhodocyclaceae & 38.73 & 51.91 & 25.07 & 0.54 & 0.92 \\
\hline Burkholderiales_incertae_sedis & 3.46 & 5.04 & 2.39 & 0.00 & 0.31 \\
\hline Comamonadaceae & 4.80 & 5.80 & 5.67 & 7.27 & 19.14 \\
\hline Burkholderiaceae & 0.11 & 0.61 & 0.60 & 0.00 & 15.01 \\
\hline Alcaligenaceae & 0.22 & 0.00 & 0.00 & 0.00 & 0.00 \\
\hline Oxalobacteraceae & 0.11 & 0.31 & 0.15 & 0.00 & 1.53 \\
\hline Nitrosomonadaceae & 0.67 & 1.53 & 0.90 & 0.00 & 0.00 \\
\hline Ferrovaceae & 0.22 & 0.31 & 0.00 & 0.00 & 0.00 \\
\hline Neisseriaceae & 0.00 & 0.00 & 0.15 & 0.43 & 0.00 \\
\hline Enterobacteriaceae & 12.17 & 0.00 & 15.22 & 0.98 & 0.92 \\
\hline Pseudomonadaceae & 0.56 & 0.00 & 0.90 & 0.00 & 1.53 \\
\hline Moraxellaceae & 0.33 & 0.00 & 0.15 & 10.09 & 0.61 \\
\hline Xanthomonadaceae & 0.22 & 0.15 & 0.60 & 0.22 & 0.46 \\
\hline Sinobacteraceae & 0.11 & 0.00 & 0.15 & 0.00 & 0.00 \\
\hline Aeromonadaceae & 0.45 & 0.00 & 0.45 & 4.77 & 1.23 \\
\hline Succinivibrionaceae & 0.00 & 0.00 & 0.30 & 0.00 & 0.00 \\
\hline Halomonadaceae & 0.00 & 0.00 & 0.00 & 0.00 & 1.23 \\
\hline Shewanellaceae & 0.00 & 0.00 & 0.00 & 0.00 & 1.68 \\
\hline Bradyrhizobiaceae & 0.89 & 0.00 & 0.60 & 0.00 & 0.15 \\
\hline Rhizobiales_incertae_sedis & 0.11 & 0.00 & 0.00 & 0.00 & 0.00 \\
\hline Methylocystaceae & 0.11 & 0.15 & 0.00 & 0.00 & 0.00 \\
\hline Hyphomicrobiaceae & 0.11 & 0.00 & 0.15 & 0.00 & 0.00 \\
\hline Phyllobacteriaceae & 0.00 & 0.00 & 0.15 & 0.00 & 0.31 \\
\hline Rhodobacteraceae & 3.46 & 0.15 & 1.34 & 0.22 & 1.23 \\
\hline Sphingomonadaceae & 0.89 & 0.61 & 0.30 & 0.00 & 0.61 \\
\hline Acetobacteraceae & 0.11 & 0.00 & 0.15 & 0.00 & 0.00 \\
\hline Rhodospirillaceae & 0.22 & 0.00 & 0.00 & 0.00 & 0.15 \\
\hline Caulobacteraceae & 0.11 & 0.00 & 0.15 & 0.00 & 2.45 \\
\hline Polyangiaceae & 0.89 & 3.05 & 0.75 & 0.00 & 0.00 \\
\hline Nannocystaceae & 1.00 & 0.15 & 1.34 & 0.00 & 0.00 \\
\hline Cystobacteraceae & 0.11 & 0.00 & 0.15 & 0.00 & 0.00 \\
\hline Bdellovibrionaceae & 0.22 & 0.46 & 0.30 & 0.00 & 0.00 \\
\hline Desulfomicrobiaceae & 0.00 & 0.15 & 0.00 & 0.00 & 0.00 \\
\hline Desulfovibrionaceae & 0.00 & 0.00 & 0.00 & 0.11 & 0.00 \\
\hline Campylobacteraceae & 0.00 & 0.15 & 1.79 & 1.63 & 0.00 \\
\hline Anaerolineaceae & 1.79 & 3.36 & 3.28 & 0.00 & 0.00 \\
\hline Caldilineaceae & 0.22 & 0.61 & 0.60 & 0.00 & 0.31 \\
\hline
\end{tabular}


Table S6B. Continued.

\begin{tabular}{|c|c|c|c|c|c|}
\hline Chloroflexaceae & 0.00 & 0.00 & 0.15 & 0.00 & 0.00 \\
\hline Leptotrichiaceae & 0.00 & 0.15 & 0.00 & 0.65 & 0.15 \\
\hline Fusobacteriaceae & 0.00 & 0.00 & 0.30 & 0.00 & 0.00 \\
\hline Flavobacteriaceae & 0.22 & 0.31 & 0.30 & 0.98 & 4.59 \\
\hline Chitinophagaceae & 0.56 & 0.76 & 0.30 & 0.00 & 0.61 \\
\hline Porphyromonadaceae & 0.11 & 0.15 & 0.00 & 0.33 & 0.00 \\
\hline Prevotellaceae & 0.00 & 0.00 & 1.34 & 38.07 & 0.00 \\
\hline Bacteroidaceae & 0.00 & 0.00 & 0.45 & 0.43 & 0.00 \\
\hline Cytophagaceae & 0.00 & 0.00 & 0.45 & 0.00 & 0.00 \\
\hline Nitrospiraceae & 4.35 & 1.53 & 4.63 & 0.00 & 0.00 \\
\hline Iamiaceae & 0.45 & 0.15 & 0.45 & 0.00 & 0.15 \\
\hline Acidimicrobiaceae & 0.22 & 0.00 & 0.30 & 0.00 & 0.00 \\
\hline Intrasporangiaceae & 1.56 & 0.61 & 2.09 & 0.00 & 0.00 \\
\hline Micrococcaceae & 0.00 & 0.00 & 0.00 & 0.00 & 0.46 \\
\hline Microbacteriaceae & 0.00 & 0.00 & 0.00 & 0.00 & 0.15 \\
\hline Nocardioidaceae & 0.33 & 0.31 & 0.60 & 0.00 & 1.23 \\
\hline Propionibacteriaceae & 0.00 & 0.00 & 0.00 & 0.33 & 0.15 \\
\hline Mycobacteriaceae & 0.11 & 0.00 & 0.00 & 0.00 & 0.00 \\
\hline Nocardiaceae & 0.00 & 0.00 & 0.15 & 0.00 & 0.15 \\
\hline Corynebacteriaceae & 0.00 & 0.00 & 0.00 & 0.00 & 0.46 \\
\hline Micromonosporaceae & 0.00 & 0.00 & 0.00 & 0.00 & 0.15 \\
\hline Nakamurellaceae & 0.00 & 0.00 & 0.00 & 0.00 & 0.15 \\
\hline Geodermatophilaceae & 0.00 & 0.00 & 0.00 & 0.00 & 0.15 \\
\hline Bifidobacteriaceae & 0.11 & 0.00 & 0.15 & 0.00 & 0.00 \\
\hline Conexibacteraceae & 0.22 & 0.00 & 0.30 & 0.00 & 0.00 \\
\hline Coriobacteriaceae & 0.00 & 0.00 & 0.00 & 0.11 & 0.00 \\
\hline Verrucomicrobiaceae & 0.56 & 0.46 & 0.30 & 0.00 & 0.00 \\
\hline Opitutaceae & 0.67 & 2.29 & 0.15 & 0.00 & 0.00 \\
\hline Lachnospiraceae & 0.22 & 0.00 & 0.45 & 3.90 & 0.61 \\
\hline Clostridiaceae 1 & 0.45 & 0.61 & 0.90 & 0.76 & 15.62 \\
\hline Peptostreptococcaceae & 1.67 & 1.07 & 2.84 & 0.43 & 8.58 \\
\hline Ruminococcaceae & 0.22 & 0.31 & 0.30 & 2.82 & 1.38 \\
\hline Clostridiales_Incertae Sedis XIII & 0.11 & 0.00 & 0.00 & 0.00 & 0.00 \\
\hline Clostridiales_Incertae Sedis XI & 0.00 & 0.00 & 0.15 & 0.00 & 0.00 \\
\hline Streptococcaceae & 0.11 & 0.00 & 0.15 & 0.00 & 0.15 \\
\hline Enterococcaceae & 0.11 & 0.00 & 0.00 & 0.00 & 0.00 \\
\hline Leuconostocaceae & 0.00 & 0.00 & 0.00 & 0.00 & 0.15 \\
\hline Bacillaceae 1 & 0.00 & 0.00 & 0.15 & 0.00 & 1.07 \\
\hline Bacillaceae 2 & 0.00 & 0.00 & 0.00 & 0.00 & 0.31 \\
\hline Erysipelotrichaceae & 0.11 & 0.15 & 0.15 & 0.22 & 1.53 \\
\hline
\end{tabular}


Table S6B. Continued.

\begin{tabular}{|c|c|c|c|c|c|}
\hline Acidaminococcaceae & 0.00 & 0.00 & 0.15 & 0.43 & 0.00 \\
\hline Veillonellaceae & 0.00 & 0.00 & 0.45 & 0.65 & 0.00 \\
\hline Deinococcaceae & 0.00 & 0.00 & 0.00 & 0.00 & 0.15 \\
\hline Fibrobacteraceae & 0.00 & 0.15 & 0.15 & 1.41 & 0.00 \\
\hline Armatimonadaceae & 0.00 & 0.15 & 0.00 & 0.00 & 0.00 \\
\hline Elusimicrobiaceae & 0.00 & 0.00 & 0.15 & 0.00 & 0.00 \\
\hline Spirochaetaceae & 0.00 & 0.00 & 0.15 & 0.00 & 0.00 \\
\hline Leptospiraceae & 0.00 & 0.00 & 0.15 & 0.00 & 0.00 \\
\hline Victivallaceae & 0.00 & 0.00 & 0.00 & 0.11 & 0.00 \\
\hline unclassified_Burkholderiales & 4.46 & 5.80 & 4.93 & 0.11 & 3.06 \\
\hline unclassified_Nitrosomonadales & 0.11 & 0.00 & 0.00 & 0.00 & 0.00 \\
\hline unclassified_Pseudomonadales & 0.45 & 0.46 & 0.90 & 0.65 & 0.15 \\
\hline unclassified_Alteromonadales & 0.00 & 0.00 & 0.00 & 0.00 & 0.31 \\
\hline unclassified_Rhizobiales & 1.23 & 0.31 & 1.64 & 0.11 & 0.77 \\
\hline unclassified_Sphingomonadales & 0.11 & 0.15 & 0.15 & 0.00 & 0.00 \\
\hline unclassified_Myxococcales & 0.33 & 0.15 & 0.15 & 0.00 & 0.00 \\
\hline unclassified_"Fusobacteriales" & 0.22 & 0.15 & 0.00 & 2.28 & 0.00 \\
\hline unclassified_"Flavobacteriales" & 0.45 & 0.00 & 0.45 & 0.11 & 0.00 \\
\hline unclassified_"Sphingobacteriales" & 0.11 & 0.00 & 0.15 & 0.00 & 0.15 \\
\hline unclassified_"Bacteroidales" & 0.11 & 0.15 & 0.75 & 15.18 & 0.00 \\
\hline unclassified_"Acidimicrobineae" & 0.56 & 0.15 & 0.15 & 0.00 & 0.15 \\
\hline unclassified_Micrococcineae & 0.22 & 0.15 & 0.60 & 0.00 & 0.31 \\
\hline unclassified_Corynebacterineae & 0.22 & 0.15 & 0.15 & 0.00 & 1.68 \\
\hline unclassified_Actinomycetales & 1.45 & 1.37 & 2.54 & 0.33 & 2.14 \\
\hline unclassified_Clostridiales & 0.33 & 1.07 & 2.09 & 2.93 & 2.91 \\
\hline unclassified_Bacillales & 0.00 & 0.00 & 0.00 & 0.00 & 0.31 \\
\hline unclassified_Selenomonadales & 0.00 & 0.00 & 0.00 & 0.43 & 0.00 \\
\hline unclassified_Cystobacterineae & 0.11 & 0.00 & 0.00 & 0.00 & 0.00 \\
\hline unclassified_Nannocystineae & 0.22 & 0.15 & 0.00 & 0.00 & 0.00 \\
\hline unclassified_Sorangiineae & 0.00 & 0.15 & 0.00 & 0.00 & 0.00 \\
\hline
\end{tabular}


Table S7A. Abundances of all genus in each sample of the Failed EBPR. The abundance is presented in terms of percentages of the total sequences in a sample.

\begin{tabular}{|c|c|c|c|c|c|}
\hline Name & Aerobic_A & Anaerobic_A & Anoxic_A & Fermenter_A & PST_A \\
\hline Acidovorax & 26.20 & 0.50 & 0.78 & 3.72 & 17.05 \\
\hline Delftia & 1.41 & 0.00 & 0.00 & 0.00 & 1.14 \\
\hline Alicycliphilus & 0.56 & 0.50 & 0.31 & 0.78 & 0.38 \\
\hline Comamonas & 0.28 & 0.33 & 0.00 & 0.16 & 0.00 \\
\hline Caenimonas & 0.00 & 0.17 & 0.78 & 0.00 & 0.19 \\
\hline Albidiferax & 0.00 & 0.17 & 0.00 & 0.00 & 0.00 \\
\hline Curvibacter & 0.00 & 0.33 & 0.00 & 0.00 & 0.00 \\
\hline Ramlibacter & 0.00 & 0.00 & 0.00 & 0.00 & 0.19 \\
\hline Chitinimonas & 0.56 & 0.00 & 0.31 & 0.00 & 0.00 \\
\hline Pandoraea & 3.38 & 0.00 & 0.00 & 0.00 & 10.61 \\
\hline Ralstonia & 0.28 & 0.00 & 0.00 & 0.00 & 0.19 \\
\hline Cupriavidus & 0.28 & 0.00 & 0.00 & 0.00 & 1.52 \\
\hline Burkholderia & 0.00 & 0.00 & 0.00 & 0.00 & 0.19 \\
\hline Massilia & 1.41 & 0.00 & 0.00 & 0.00 & 1.14 \\
\hline Naxibacter & 0.56 & 0.00 & 0.00 & 0.00 & 0.00 \\
\hline Noviherbaspirillum & 0.00 & 0.00 & 0.00 & 0.00 & 0.57 \\
\hline Aquincola & 0.28 & 0.00 & 0.00 & 0.00 & 0.19 \\
\hline Ideonella & 0.00 & 0.17 & 0.00 & 0.00 & 0.00 \\
\hline Rivibacter & 0.00 & 0.17 & 0.00 & 0.00 & 0.00 \\
\hline Inhella & 0.00 & 0.17 & 0.78 & 0.00 & 0.00 \\
\hline Rubrivivax & 0.00 & 0.00 & 0.16 & 0.00 & 0.00 \\
\hline Xylophilus & 0.00 & 0.00 & 0.16 & 0.00 & 0.00 \\
\hline Aquabacterium & 0.00 & 0.00 & 0.16 & 0.00 & 0.19 \\
\hline Derxia & 0.00 & 0.00 & 0.47 & 0.00 & 0.00 \\
\hline Alcaligenes & 0.00 & 0.00 & 0.00 & 0.00 & 0.19 \\
\hline Propionivibrio & 0.28 & 4.17 & 5.49 & 0.00 & 0.19 \\
\hline Ferribacterium & 0.00 & 0.33 & 0.31 & 0.00 & 0.00 \\
\hline Sulfuritalea & 0.00 & 1.67 & 0.94 & 0.00 & 0.00 \\
\hline Methyloversatilis & 0.00 & 0.33 & 0.16 & 0.00 & 0.19 \\
\hline Zoogloea & 0.00 & 0.83 & 0.31 & 0.16 & 0.19 \\
\hline Dechloromonas & 0.00 & 0.17 & 0.16 & 0.31 & 0.00 \\
\hline Thauera & 0.00 & 0.00 & 0.16 & 0.31 & 0.00 \\
\hline Rhodocyclus & 0.00 & 0.00 & 0.31 & 0.00 & 0.00 \\
\hline Uruburuella & 0.00 & 0.33 & 0.00 & 0.00 & 0.19 \\
\hline Laribacter & 0.00 & 0.17 & 0.00 & 0.31 & 0.00 \\
\hline Ferrovum & 0.00 & 0.83 & 0.00 & 0.00 & 0.00 \\
\hline Mesorhizobium & 0.00 & 0.17 & 0.00 & 0.00 & 0.00 \\
\hline Microvirga & 0.56 & 0.00 & 0.00 & 0.00 & 0.19 \\
\hline Devosia & 0.28 & 0.00 & 0.00 & 0.00 & 0.00 \\
\hline
\end{tabular}


Table S7A. Continued

\begin{tabular}{|c|c|c|c|c|c|}
\hline Prosthecomicrobium & 0.00 & 0.00 & 0.31 & 0.00 & 0.00 \\
\hline Aquabacter & 0.00 & 0.00 & 0.00 & 0.16 & 0.00 \\
\hline Hyphomicrobium & 0.00 & 0.00 & 0.00 & 0.00 & 0.19 \\
\hline Afipia & 0.00 & 0.17 & 0.00 & 0.00 & 0.00 \\
\hline Oligotropha & 0.00 & 0.50 & 0.16 & 0.00 & 0.00 \\
\hline Brevundimonas & 6.76 & 0.00 & 0.00 & 0.00 & 4.36 \\
\hline Phenylobacterium & 0.28 & 0.17 & 0.00 & 0.00 & 0.19 \\
\hline Sphingomonas & 0.28 & 0.00 & 0.00 & 0.00 & 0.76 \\
\hline Sphingorhabdus & 0.00 & 0.17 & 0.00 & 0.00 & 0.00 \\
\hline Pannonibacter & 0.56 & 0.00 & 0.00 & 0.00 & 0.38 \\
\hline Amaricoccus & 0.28 & 0.00 & 0.00 & 0.16 & 0.19 \\
\hline Gemmobacter & 0.00 & 0.00 & 0.16 & 0.00 & 0.19 \\
\hline Paracoccus & 0.00 & 0.00 & 0.00 & 0.47 & 0.38 \\
\hline Roseomonas & 0.28 & 0.00 & 0.00 & 0.00 & 0.00 \\
\hline Stella & 0.00 & 0.17 & 0.00 & 0.00 & 0.00 \\
\hline Magnetospirillum & 0.00 & 0.17 & 0.00 & 0.00 & 0.00 \\
\hline Defluviicoccus & 0.00 & 0.17 & 0.00 & 0.00 & 0.00 \\
\hline Azospirillum & 0.00 & 0.00 & 0.00 & 0.00 & 0.19 \\
\hline Desertibacter & 0.00 & 0.00 & 0.00 & 0.00 & 0.38 \\
\hline Enterobacter & 0.28 & 0.00 & 0.00 & 0.00 & 0.00 \\
\hline Proteus & 0.00 & 0.00 & 0.00 & 0.31 & 0.19 \\
\hline Citrobacter & 0.00 & 0.00 & 0.00 & 0.16 & 0.00 \\
\hline Pseudomonas & 0.56 & 0.00 & 0.00 & 0.31 & 0.19 \\
\hline Cellvibrio & 0.00 & 1.00 & 1.73 & 0.00 & 0.00 \\
\hline Serpens & 0.00 & 0.00 & 0.16 & 0.00 & 0.19 \\
\hline Acinetobacter & 0.56 & 0.67 & 0.31 & 11.94 & 0.57 \\
\hline Enhydrobacter & 0.00 & 0.33 & 0.31 & 2.79 & 0.57 \\
\hline Alkanindiges & 0.00 & 0.00 & 0.00 & 0.31 & 0.19 \\
\hline Halomonas & 0.28 & 0.00 & 0.00 & 0.00 & 0.38 \\
\hline Shewanella & 0.85 & 0.00 & 0.00 & 0.00 & 0.76 \\
\hline Lysobacter & 0.28 & 0.00 & 0.00 & 0.00 & 0.00 \\
\hline Dokdonella & 0.00 & 0.50 & 0.78 & 0.00 & 0.00 \\
\hline Thermomonas & 0.00 & 0.00 & 0.16 & 0.00 & 0.00 \\
\hline Aquimonas & 0.00 & 0.00 & 0.31 & 0.00 & 0.00 \\
\hline Aeromonas & 0.28 & 0.50 & 1.10 & 8.53 & 0.19 \\
\hline Tolumonas & 0.00 & 0.50 & 0.31 & 2.48 & 0.00 \\
\hline Rheinheimera & 0.00 & 0.00 & 0.00 & 0.00 & 0.19 \\
\hline Thiothrix & 0.00 & 0.00 & 0.00 & 0.00 & 0.19 \\
\hline Arcobacter & 0.28 & 1.17 & 0.16 & 6.98 & 0.38 \\
\hline Sulfurospirillum & 0.00 & 0.00 & 0.00 & 0.16 & 0.00 \\
\hline Byssovorax & 0.00 & 0.83 & 0.16 & 0.00 & 0.00 \\
\hline
\end{tabular}


Table S7A. Continued

\begin{tabular}{|c|c|c|c|c|c|}
\hline Nannocystis & 0.00 & 2.50 & 2.35 & 0.00 & 0.00 \\
\hline Haliangium & 0.00 & 0.17 & 0.00 & 0.00 & 0.00 \\
\hline Kofleria & 0.00 & 0.17 & 0.16 & 0.00 & 0.00 \\
\hline Bacteriovorax & 0.00 & 0.00 & 0.00 & 0.00 & 0.19 \\
\hline Vampirovibrio & 0.00 & 0.00 & 0.00 & 0.00 & 0.19 \\
\hline Blastococcus & 0.28 & 0.00 & 0.00 & 0.00 & 0.95 \\
\hline Mycobacterium & 0.00 & 0.00 & 0.63 & 0.00 & 0.00 \\
\hline Corynebacterium & 0.00 & 0.00 & 0.00 & 0.00 & 0.19 \\
\hline Microbacterium & 0.28 & 0.00 & 0.00 & 0.00 & 0.19 \\
\hline Leucobacter & 0.28 & 0.00 & 0.00 & 0.00 & 0.00 \\
\hline Agrococcus & 0.00 & 0.00 & 0.00 & 0.00 & 0.19 \\
\hline Tetrasphaera & 0.00 & 1.34 & 0.63 & 0.00 & 0.00 \\
\hline Dermacoccus & 0.00 & 0.17 & 0.00 & 0.00 & 0.00 \\
\hline Nesterenkonia & 0.00 & 0.00 & 0.00 & 0.00 & 0.19 \\
\hline Nocardioides & 0.00 & 0.17 & 0.16 & 0.00 & 0.00 \\
\hline Marmoricola & 0.00 & 0.33 & 0.47 & 0.00 & 0.57 \\
\hline Pimelobacter & 0.00 & 0.17 & 0.00 & 0.00 & 0.00 \\
\hline Brooklawnia & 0.00 & 0.00 & 0.00 & 0.16 & 0.00 \\
\hline Micropruina & 0.00 & 0.00 & 0.00 & 0.00 & 0.19 \\
\hline Actinomyces & 0.28 & 0.00 & 0.00 & 0.00 & 0.19 \\
\hline Bifidobacterium & 0.00 & 0.33 & 0.00 & 0.16 & 0.00 \\
\hline Collinsella & 0.28 & 0.00 & 0.00 & 0.00 & 0.00 \\
\hline Iamia & 0.00 & 1.00 & 0.00 & 0.00 & 0.00 \\
\hline Ilumatobacter & 0.00 & 0.33 & 0.16 & 0.00 & 0.00 \\
\hline Streptococcus & 0.28 & 0.00 & 0.16 & 0.47 & 0.00 \\
\hline Aeribacillus & 0.00 & 0.00 & 0.00 & 0.00 & 0.57 \\
\hline Turicibacter & 0.56 & 0.00 & 0.47 & 0.00 & 0.38 \\
\hline Erysipelotrichaceae_incertae_sedis & 0.00 & 0.00 & 0.16 & 0.00 & 0.00 \\
\hline Clostridium XI & 3.66 & 1.34 & 0.94 & 0.31 & 1.89 \\
\hline Proteocatella & 0.00 & 0.00 & 0.16 & 0.00 & 0.00 \\
\hline Peptostreptococcaceae_incertae_sedis & 0.00 & 0.00 & 0.00 & 0.00 & 0.19 \\
\hline Clostridium sensu stricto & 2.82 & 0.33 & 0.31 & 0.47 & 1.89 \\
\hline Anaerobacter & 0.00 & 0.17 & 0.00 & 0.00 & 0.00 \\
\hline Sarcina & 0.00 & 0.00 & 0.16 & 0.00 & 0.00 \\
\hline Faecalibacterium & 0.28 & 0.00 & 0.00 & 1.09 & 0.57 \\
\hline Oscillibacter & 0.00 & 0.00 & 0.00 & 0.31 & 0.00 \\
\hline Subdoligranulum & 0.00 & 0.00 & 0.00 & 0.16 & 0.00 \\
\hline Saccharofermentans & 0.00 & 0.00 & 0.00 & 0.00 & 0.38 \\
\hline Lachnospiracea_incertae_sedis & 0.00 & 0.17 & 0.00 & 0.00 & 0.00 \\
\hline Ruminococcus2 & 0.00 & 0.17 & 0.00 & 0.00 & 0.19 \\
\hline Roseburia & 0.00 & 0.00 & 0.00 & 0.16 & 0.00 \\
\hline
\end{tabular}


Table S7A. Continued

\begin{tabular}{|c|c|c|c|c|c|}
\hline Blautia & 0.00 & 0.00 & 0.00 & 0.00 & 0.19 \\
\hline Phascolarctobacterium & 0.00 & 0.00 & 0.00 & 0.16 & 0.00 \\
\hline Succinispira & 0.00 & 0.00 & 0.00 & 0.16 & 0.00 \\
\hline Megasphaera & 0.00 & 0.00 & 0.16 & 0.16 & 0.00 \\
\hline Mitsuokella & 0.00 & 0.00 & 0.00 & 0.47 & 0.00 \\
\hline Parcubacteria_genera_incertae_sedis & 1.69 & 0.17 & 0.00 & 0.16 & 3.60 \\
\hline Flavobacterium & 3.94 & 0.50 & 0.94 & 0.47 & 3.03 \\
\hline Cloacibacterium & 0.00 & 0.00 & 0.16 & 1.09 & 0.38 \\
\hline Niastella & 0.28 & 0.00 & 0.00 & 0.00 & 0.00 \\
\hline Terrimonas & 0.00 & 0.33 & 0.16 & 0.00 & 0.00 \\
\hline Sediminibacterium & 0.00 & 0.17 & 0.47 & 0.00 & 0.00 \\
\hline Ferruginibacter & 0.00 & 0.17 & 0.00 & 0.00 & 0.00 \\
\hline Haliscomenobacter & 0.00 & 0.33 & 0.47 & 0.00 & 0.00 \\
\hline Arcticibacter & 0.00 & 0.00 & 0.16 & 0.00 & 0.00 \\
\hline Pedobacter & 0.00 & 0.00 & 0.16 & 0.00 & 0.00 \\
\hline Leadbetterella & 0.00 & 0.33 & 0.00 & 0.00 & 0.00 \\
\hline Runella & 0.00 & 0.33 & 0.00 & 0.00 & 0.00 \\
\hline Ohtaekwangia & 0.00 & 0.17 & 0.00 & 0.00 & 0.00 \\
\hline Prevotella & 0.00 & 0.17 & 0.16 & 3.72 & 0.00 \\
\hline Xylanibacter & 0.00 & 0.00 & 0.00 & 3.57 & 0.00 \\
\hline Bacteroides & 0.00 & 0.00 & 0.00 & 0.62 & 0.00 \\
\hline Saccharibacteria_genera_incertae_sedis & 1.13 & 0.00 & 0.16 & 0.00 & 6.06 \\
\hline Longilinea & 0.00 & 0.17 & 0.00 & 0.00 & 0.00 \\
\hline Litorilinea & 0.00 & 0.17 & 0.00 & 0.00 & 0.00 \\
\hline Caldilinea & 0.00 & 0.17 & 0.16 & 0.00 & 0.19 \\
\hline Roseiflexus & 0.00 & 0.17 & 0.00 & 0.00 & 0.00 \\
\hline Herpetosiphon & 0.00 & 0.00 & 0.00 & 0.00 & 0.19 \\
\hline Nitrospira & 0.00 & 3.34 & 2.67 & 0.00 & 0.00 \\
\hline Gemmata & 0.00 & 0.67 & 0.47 & 0.00 & 0.00 \\
\hline Planctomyces & 0.00 & 0.50 & 0.16 & 0.00 & 0.00 \\
\hline Aquisphaera & 0.00 & 0.00 & 0.16 & 0.00 & 0.00 \\
\hline Prosthecobacter & 0.00 & 0.67 & 0.00 & 0.00 & 0.19 \\
\hline Luteolibacter & 0.00 & 0.17 & 0.00 & 0.00 & 0.00 \\
\hline Subdivision3_genera_incertae_sedis & 0.00 & 0.83 & 0.16 & 0.00 & 0.00 \\
\hline Opitutus & 0.00 & 1.00 & 1.57 & 0.31 & 0.00 \\
\hline Blastocatella & 0.00 & 0.17 & 0.00 & 0.00 & 0.00 \\
\hline Turneriella & 0.00 & 0.67 & 0.00 & 0.00 & 0.00 \\
\hline Armatimonadetes_gp5 & 0.00 & 0.17 & 0.00 & 0.00 & 0.00 \\
\hline Fimbriimonas & 0.00 & 0.00 & 0.16 & 0.00 & 0.00 \\
\hline Gemmatimonas & 0.00 & 0.00 & 0.16 & 0.00 & 0.00 \\
\hline Victivallis & 0.00 & 0.00 & 0.00 & 0.16 & 0.00 \\
\hline
\end{tabular}


Table S7A. Continued

\begin{tabular}{|c|c|c|c|c|c|}
\hline Bacillariophyta & 0.00 & 0.00 & 0.00 & 0.16 & 0.00 \\
\hline Elusimicrobium & 0.00 & 0.00 & 0.00 & 0.00 & 0.19 \\
\hline unclassified_Comamonadaceae & 13.52 & 3.67 & 3.61 & 9.15 & 9.47 \\
\hline unclassified_Oxalobacteraceae & 0.56 & 0.00 & 0.00 & 0.00 & 1.52 \\
\hline unclassified_Burkholderiales_incertae_sedis & 1.13 & 2.50 & 2.20 & 0.00 & 0.95 \\
\hline unclassified_Burkholderiaceae & 0.28 & 0.00 & 0.00 & 0.00 & 0.19 \\
\hline unclassified_Rhodocyclaceae & 0.85 & 29.22 & 35.79 & 1.40 & 0.95 \\
\hline unclassified_Neisseriaceae & 0.00 & 0.00 & 0.00 & 0.47 & 0.00 \\
\hline unclassified_Nitrosomonadaceae & 0.00 & 0.83 & 1.26 & 0.00 & 0.38 \\
\hline unclassified_Phyllobacteriaceae & 0.28 & 0.17 & 0.31 & 0.00 & 0.76 \\
\hline unclassified_Bradyrhizobiaceae & 0.00 & 0.33 & 0.94 & 0.00 & 0.19 \\
\hline unclassified_Rhizobiaceae & 0.00 & 0.17 & 0.00 & 0.00 & 0.00 \\
\hline unclassified_Methylocystaceae & 0.00 & 0.00 & 0.16 & 0.00 & 0.00 \\
\hline unclassified_Beijerinckiaceae & 0.00 & 0.00 & 0.00 & 0.00 & 0.19 \\
\hline unclassified_Caulobacteraceae & 0.56 & 0.00 & 0.00 & 0.00 & 0.76 \\
\hline unclassified_Sphingomonadaceae & 0.56 & 0.00 & 0.47 & 0.00 & 0.95 \\
\hline unclassified_Erythrobacteraceae & 0.00 & 0.00 & 0.00 & 0.00 & 0.19 \\
\hline unclassified_Rhodobacteraceae & 0.28 & 1.50 & 2.20 & 0.31 & 0.38 \\
\hline unclassified_Acetobacteraceae & 0.00 & 0.17 & 0.31 & 0.00 & 0.00 \\
\hline unclassified_Rhodospirillaceae & 0.00 & 0.00 & 0.47 & 0.00 & 0.19 \\
\hline unclassified_Enterobacteriaceae & 3.66 & 0.33 & 0.47 & 2.33 & 0.95 \\
\hline unclassified_Pseudomonadaceae & 5.63 & 0.00 & 0.16 & 0.78 & 3.79 \\
\hline unclassified_Moraxellaceae & 0.00 & 0.50 & 0.47 & 3.57 & 0.57 \\
\hline unclassified_Halomonadaceae & 0.00 & 0.00 & 0.00 & 0.00 & 0.95 \\
\hline unclassified_Xanthomonadaceae & 0.28 & 0.33 & 1.26 & 0.00 & 0.19 \\
\hline unclassified_Aeromonadaceae & 0.00 & 0.17 & 0.16 & 0.93 & 0.00 \\
\hline unclassified_Polyangiaceae & 0.00 & 1.34 & 0.47 & 0.00 & 0.00 \\
\hline unclassified_Nannocystaceae & 0.00 & 1.84 & 0.94 & 0.00 & 0.00 \\
\hline unclassified_Nocardiaceae & 0.28 & 0.17 & 0.00 & 0.00 & 0.00 \\
\hline unclassified_Microbacteriaceae & 0.56 & 0.17 & 0.00 & 0.00 & 0.38 \\
\hline unclassified_Nocardioidaceae & 0.28 & 0.33 & 0.00 & 0.00 & 0.38 \\
\hline unclassified_Propionibacteriaceae & 0.00 & 0.00 & 0.16 & 0.00 & 0.19 \\
\hline unclassified_Acidimicrobiaceae & 0.00 & 0.00 & 0.31 & 0.00 & 0.00 \\
\hline unclassified_Streptococcaceae & 0.00 & 0.00 & 0.00 & 0.00 & 0.19 \\
\hline unclassified_Clostridiaceae 1 & 3.38 & 1.17 & 1.10 & 0.62 & 1.14 \\
\hline unclassified_Peptostreptococcaceae & 0.85 & 0.33 & 0.47 & 0.47 & 2.08 \\
\hline unclassified_Ruminococcaceae & 0.56 & 0.33 & 0.16 & 0.47 & 0.57 \\
\hline unclassified_Lachnospiraceae & 0.00 & 0.67 & 0.47 & 0.62 & 0.57 \\
\hline unclassified_Acidaminococcaceae & 0.00 & 0.17 & 0.00 & 0.62 & 0.00 \\
\hline unclassified_Veillonellaceae & 0.00 & 0.00 & 0.00 & 0.31 & 0.00 \\
\hline unclassified_Flavobacteriaceae & 1.97 & 0.67 & 0.63 & 0.78 & 0.76 \\
\hline
\end{tabular}


Table S7A. Continued.

\begin{tabular}{|c|c|c|c|c|c|}
\hline unclassified_Chitinophagaceae & 0.00 & 1.00 & 0.63 & 0.00 & 0.00 \\
\hline unclassified_"Prevotellaceae" & 0.00 & 0.50 & 0.00 & 19.53 & 0.00 \\
\hline unclassified_"Porphyromonadaceae" & 0.00 & 0.00 & 0.00 & 0.31 & 0.00 \\
\hline unclassified_"Leptotrichiaceae” & 0.00 & 0.17 & 0.16 & 1.24 & 0.00 \\
\hline unclassified_"Fusobacteriaceae” & 0.00 & 0.00 & 0.16 & 1.24 & 0.19 \\
\hline unclassified_Anaerolineaceae & 0.28 & 9.68 & 9.26 & 0.00 & 0.76 \\
\hline unclassified_Caldilineaceae & 0.00 & 0.50 & 0.00 & 0.00 & 0.38 \\
\hline unclassified_Chloroflexaceae & 0.00 & 0.17 & 0.00 & 0.00 & 0.00 \\
\hline unclassified_Planctomycetaceae & 0.00 & 1.34 & 1.26 & 0.00 & 0.00 \\
\hline unclassified_Verrucomicrobiaceae & 0.00 & 0.00 & 0.16 & 0.00 & 0.00 \\
\hline unclassified_Opitutaceae & 0.00 & 0.33 & 0.00 & 0.00 & 0.00 \\
\hline unclassified_Holophagaceae & 0.00 & 0.33 & 0.00 & 0.00 & 0.00 \\
\hline
\end{tabular}

Table S7B. Abundances of all genus in each sample of the Successful EBPR. The abundance is presented in terms of percentages of the total sequences in a sample.

\begin{tabular}{|c|c|c|c|c|c|}
\hline Name & Aerobic_B & Anaerobic_B & Anoxic_B & Fermenter_B & PST_B \\
\hline Planctomyces & 0.62 & 1.68 & 0.70 & 0.00 & 0.17 \\
\hline Schlesneria & 0.12 & 0.17 & 0.00 & 0.00 & 0.00 \\
\hline Pirellula & 0.12 & 0.17 & 0.17 & 0.00 & 0.00 \\
\hline Gemmata & 0.00 & 0.17 & 0.52 & 0.00 & 0.00 \\
\hline Phycisphaera & 0.00 & 0.17 & 0.00 & 0.00 & 0.00 \\
\hline Sulfuritalea & 1.36 & 0.51 & 0.17 & 0.00 & 0.00 \\
\hline Propionivibrio & 3.45 & 8.59 & 2.61 & 0.00 & 0.34 \\
\hline Methyloversatilis & 0.25 & 0.00 & 0.17 & 0.00 & 0.00 \\
\hline Dechloromonas & 0.12 & 0.17 & 0.35 & 0.14 & 0.17 \\
\hline Ferribacterium & 0.12 & 0.00 & 0.35 & 0.00 & 0.00 \\
\hline Zoogloea & 0.12 & 0.17 & 0.17 & 0.56 & 0.00 \\
\hline Thauera & 0.12 & 0.17 & 0.00 & 0.00 & 0.00 \\
\hline Piscinibacter & 0.12 & 0.00 & 0.00 & 0.00 & 0.00 \\
\hline Aquabacterium & 0.49 & 0.34 & 0.17 & 0.00 & 0.00 \\
\hline Inhella & 0.49 & 0.67 & 0.17 & 0.00 & 0.00 \\
\hline Ideonella & 0.00 & 0.17 & 0.17 & 0.00 & 0.00 \\
\hline Rubrivivax & 0.00 & 0.00 & 0.17 & 0.00 & 0.00 \\
\hline Caenimonas & 1.36 & 1.01 & 1.05 & 0.00 & 0.00 \\
\hline Ottowia & 0.12 & 0.00 & 0.00 & 0.00 & 0.00 \\
\hline Simplicispira & 0.12 & 0.17 & 0.00 & 0.00 & 0.00 \\
\hline Comamonas & 0.37 & 0.00 & 0.17 & 0.56 & 1.02 \\
\hline Rhodoferax & 0.12 & 0.00 & 0.00 & 0.00 & 0.00 \\
\hline Alicycliphilus & 0.12 & 0.00 & 0.35 & 0.28 & 0.00 \\
\hline Albidiferax & 0.12 & 0.00 & 0.00 & 0.00 & 0.00 \\
\hline
\end{tabular}


Table S7B. Continued

\begin{tabular}{|c|c|c|c|c|c|}
\hline Curvibacter & 0.12 & 0.17 & 0.17 & 0.00 & 0.00 \\
\hline Acidovorax & 0.12 & 0.51 & 0.17 & 2.36 & 13.44 \\
\hline Delftia & 0.12 & 0.00 & 0.00 & 0.00 & 0.51 \\
\hline Malikia & 0.00 & 0.00 & 0.17 & 0.00 & 0.00 \\
\hline Macromonas & 0.00 & 0.00 & 0.17 & 0.00 & 0.00 \\
\hline Pseudorhodoferax & 0.00 & 0.00 & 0.17 & 0.00 & 0.00 \\
\hline Chitinimonas & 0.12 & 0.51 & 0.70 & 0.00 & 0.00 \\
\hline Pandoraea & 0.00 & 0.00 & 0.00 & 0.00 & 14.63 \\
\hline Cupriavidus & 0.00 & 0.00 & 0.00 & 0.00 & 1.02 \\
\hline Ralstonia & 0.00 & 0.00 & 0.00 & 0.00 & 0.17 \\
\hline Derxia & 0.25 & 0.00 & 0.00 & 0.00 & 0.00 \\
\hline Undibacterium & 0.12 & 0.34 & 0.17 & 0.00 & 0.00 \\
\hline Massilia & 0.00 & 0.00 & 0.00 & 0.00 & 1.02 \\
\hline Ferrovum & 0.25 & 0.34 & 0.00 & 0.00 & 0.00 \\
\hline Uruburuella & 0.00 & 0.00 & 0.00 & 0.14 & 0.00 \\
\hline Laribacter & 0.00 & 0.00 & 0.00 & 0.28 & 0.00 \\
\hline Proteus & 6.41 & 0.00 & 4.88 & 0.14 & 0.17 \\
\hline Escherichia/Shigella & 0.12 & 0.00 & 0.52 & 0.00 & 0.00 \\
\hline Yokenella & 0.25 & 0.00 & 0.00 & 0.14 & 0.00 \\
\hline Citrobacter & 0.37 & 0.00 & 0.35 & 0.00 & 0.00 \\
\hline Morganella & 0.12 & 0.00 & 0.35 & 0.00 & 0.00 \\
\hline Enterobacter & 0.00 & 0.00 & 0.17 & 0.00 & 0.17 \\
\hline Cosenzaea & 0.00 & 0.00 & 0.35 & 0.00 & 0.00 \\
\hline Pluralibacter & 0.00 & 0.00 & 0.17 & 0.00 & 0.00 \\
\hline Serratia & 0.00 & 0.00 & 0.17 & 0.00 & 0.00 \\
\hline Rhizobacter & 0.49 & 0.00 & 0.35 & 0.00 & 0.00 \\
\hline Pseudomonas & 0.12 & 0.00 & 0.35 & 0.00 & 0.51 \\
\hline Cellvibrio & 0.00 & 0.00 & 0.17 & 0.00 & 0.00 \\
\hline Enhydrobacter & 0.25 & 0.00 & 0.17 & 1.25 & 0.34 \\
\hline Acinetobacter & 0.12 & 0.00 & 0.00 & 8.34 & 0.34 \\
\hline Dokdonella & 0.12 & 0.00 & 0.35 & 0.00 & 0.00 \\
\hline Stenotrophomonas & 0.00 & 0.00 & 0.00 & 0.14 & 0.17 \\
\hline Thermomonas & 0.00 & 0.00 & 0.00 & 0.14 & 0.00 \\
\hline Lysobacter & 0.00 & 0.00 & 0.00 & 0.00 & 0.17 \\
\hline Pseudoxanthomonas & 0.00 & 0.00 & 0.00 & 0.00 & 0.17 \\
\hline Steroidobacter & 0.12 & 0.00 & 0.17 & 0.00 & 0.00 \\
\hline Aeromonas & 0.49 & 0.00 & 0.35 & 3.62 & 1.19 \\
\hline Tolumonas & 0.00 & 0.00 & 0.17 & 1.81 & 0.00 \\
\hline Succinivibrio & 0.00 & 0.00 & 0.17 & 0.00 & 0.00 \\
\hline Halomonas & 0.00 & 0.00 & 0.00 & 0.00 & 0.17 \\
\hline Shewanella & 0.00 & 0.00 & 0.00 & 0.00 & 1.87 \\
\hline
\end{tabular}


Table S7B. Continued

\begin{tabular}{|c|c|c|c|c|c|}
\hline Rhodopseudomonas & 0.25 & 0.00 & 0.17 & 0.00 & 0.00 \\
\hline Vasilyevaea & 0.12 & 0.00 & 0.00 & 0.00 & 0.00 \\
\hline Methylocystis & 0.12 & 0.00 & 0.00 & 0.00 & 0.00 \\
\hline Prosthecomicrobium & 0.12 & 0.00 & 0.00 & 0.00 & 0.00 \\
\hline Hyphomicrobium & 0.00 & 0.00 & 0.17 & 0.00 & 0.00 \\
\hline Rhodobacter & 0.12 & 0.00 & 0.00 & 0.00 & 0.00 \\
\hline Amaricoccus & 0.00 & 0.00 & 0.17 & 0.00 & 0.17 \\
\hline Gemmobacter & 0.00 & 0.00 & 0.00 & 0.14 & 0.00 \\
\hline Paracoccus & 0.00 & 0.00 & 0.00 & 0.14 & 0.00 \\
\hline Pannonibacter & 0.00 & 0.00 & 0.00 & 0.00 & 0.34 \\
\hline Sphingomonas & 0.12 & 0.00 & 0.00 & 0.00 & 0.51 \\
\hline Sphingorhabdus & 0.12 & 0.34 & 0.17 & 0.00 & 0.00 \\
\hline Blastomonas & 0.12 & 0.00 & 0.00 & 0.00 & 0.00 \\
\hline Defluviicoccus & 0.25 & 0.00 & 0.00 & 0.00 & 0.00 \\
\hline Skermanella & 0.00 & 0.00 & 0.00 & 0.00 & 0.17 \\
\hline Caulobacter & 0.12 & 0.00 & 0.17 & 0.00 & 0.00 \\
\hline Brevundimonas & 0.00 & 0.00 & 0.00 & 0.00 & 2.21 \\
\hline Byssovorax & 0.12 & 1.18 & 0.00 & 0.00 & 0.00 \\
\hline Nannocystis & 0.49 & 0.00 & 1.22 & 0.00 & 0.00 \\
\hline Bdellovibrio & 0.25 & 0.51 & 0.35 & 0.00 & 0.00 \\
\hline Desulfomicrobium & 0.00 & 0.17 & 0.00 & 0.00 & 0.00 \\
\hline Arcobacter & 0.00 & 0.17 & 2.09 & 2.09 & 0.00 \\
\hline Caldilinea & 0.00 & 0.00 & 0.17 & 0.00 & 0.34 \\
\hline Cloacibacterium & 0.12 & 0.00 & 0.00 & 0.00 & 0.17 \\
\hline Flavobacterium & 0.00 & 0.17 & 0.17 & 0.56 & 4.08 \\
\hline Chryseobacterium & 0.00 & 0.00 & 0.00 & 0.14 & 0.00 \\
\hline Terrimonas & 0.00 & 0.51 & 0.00 & 0.00 & 0.00 \\
\hline Ferruginibacter & 0.00 & 0.34 & 0.00 & 0.00 & 0.00 \\
\hline Hydrotalea & 0.00 & 0.00 & 0.00 & 0.00 & 0.68 \\
\hline Paludibacter & 0.00 & 0.17 & 0.00 & 0.14 & 0.00 \\
\hline Parabacteroides & 0.00 & 0.00 & 0.00 & 0.14 & 0.00 \\
\hline Prevotella & 0.00 & 0.00 & 0.35 & 7.09 & 0.00 \\
\hline Xylanibacter & 0.00 & 0.00 & 0.35 & 0.83 & 0.00 \\
\hline Bacteroides & 0.00 & 0.00 & 0.52 & 0.56 & 0.00 \\
\hline Ohtaekwangia & 0.25 & 0.00 & 0.17 & 0.14 & 0.00 \\
\hline Runella & 0.00 & 0.00 & 0.35 & 0.00 & 0.00 \\
\hline Leadbetterella & 0.00 & 0.00 & 0.17 & 0.00 & 0.00 \\
\hline Nitrospira & 4.81 & 1.68 & 5.40 & 0.00 & 0.00 \\
\hline Iamia & 0.49 & 0.17 & 0.52 & 0.00 & 0.17 \\
\hline Ilumatobacter & 0.25 & 0.00 & 0.17 & 0.00 & 0.00 \\
\hline Tetrasphaera & 1.48 & 0.51 & 2.09 & 0.00 & 0.00 \\
\hline
\end{tabular}


Table S7B. Continued

\begin{tabular}{|c|c|c|c|c|c|}
\hline Nesterenkonia & 0.00 & 0.00 & 0.00 & 0.00 & 0.34 \\
\hline Micrococcus & 0.00 & 0.00 & 0.00 & 0.00 & 0.17 \\
\hline Microbacterium & 0.00 & 0.00 & 0.00 & 0.00 & 0.17 \\
\hline Marmoricola & 0.25 & 0.34 & 0.17 & 0.00 & 0.85 \\
\hline Nocardioides & 0.00 & 0.00 & 0.17 & 0.00 & 0.17 \\
\hline Tessaracoccus & 0.00 & 0.00 & 0.00 & 0.14 & 0.00 \\
\hline Micropruina & 0.00 & 0.00 & 0.00 & 0.14 & 0.00 \\
\hline Brooklawnia & 0.00 & 0.00 & 0.00 & 0.00 & 0.17 \\
\hline Mycobacterium & 0.12 & 0.00 & 0.00 & 0.00 & 0.00 \\
\hline Skermania & 0.00 & 0.00 & 0.17 & 0.00 & 0.00 \\
\hline Gordonia & 0.00 & 0.00 & 0.00 & 0.00 & 0.17 \\
\hline Corynebacterium & 0.00 & 0.00 & 0.00 & 0.00 & 0.51 \\
\hline Nakamurella & 0.00 & 0.00 & 0.00 & 0.00 & 0.17 \\
\hline Blastococcus & 0.00 & 0.00 & 0.00 & 0.00 & 0.17 \\
\hline Bifidobacterium & 0.12 & 0.00 & 0.17 & 0.00 & 0.00 \\
\hline Conexibacter & 0.25 & 0.00 & 0.35 & 0.00 & 0.00 \\
\hline Subdivision3_genera_incertae_sedis & 0.49 & 0.51 & 0.17 & 0.00 & 0.00 \\
\hline Roseimicrobium & 0.12 & 0.00 & 0.00 & 0.00 & 0.00 \\
\hline Prosthecobacter & 0.37 & 0.34 & 0.00 & 0.00 & 0.00 \\
\hline Opitutus & 0.62 & 2.36 & 0.17 & 0.00 & 0.00 \\
\hline Gp6 & 0.12 & 0.00 & 0.00 & 0.00 & 0.00 \\
\hline Gp4 & 0.00 & 0.34 & 0.00 & 0.00 & 0.00 \\
\hline Saccharibacteria_genera_incertae_sedis & 0.25 & 0.34 & 0.00 & 0.00 & 1.70 \\
\hline Blautia & 0.00 & 0.00 & 0.17 & 0.00 & 0.00 \\
\hline Coprococcus & 0.00 & 0.00 & 0.00 & 0.14 & 0.00 \\
\hline Roseburia & 0.00 & 0.00 & 0.00 & 0.14 & 0.17 \\
\hline Cellulosilyticum & 0.00 & 0.00 & 0.00 & 0.00 & 0.17 \\
\hline Sarcina & 0.12 & 0.00 & 0.17 & 0.00 & 0.00 \\
\hline Clostridium sensu stricto & 0.00 & 0.34 & 0.35 & 0.28 & 7.31 \\
\hline Proteiniclasticum & 0.00 & 0.17 & 0.00 & 0.00 & 0.00 \\
\hline Anaerobacter & 0.00 & 0.00 & 0.00 & 0.00 & 0.17 \\
\hline Clostridium XI & 1.36 & 0.67 & 2.44 & 0.28 & 6.12 \\
\hline Faecalibacterium & 0.00 & 0.00 & 0.35 & 2.09 & 0.85 \\
\hline Oscillibacter & 0.00 & 0.00 & 0.00 & 0.14 & 0.00 \\
\hline Ruminococcus & 0.00 & 0.00 & 0.00 & 0.28 & 0.00 \\
\hline Anaerofilum & 0.00 & 0.00 & 0.00 & 0.14 & 0.00 \\
\hline Mogibacterium & 0.12 & 0.00 & 0.00 & 0.00 & 0.00 \\
\hline Gallicola & 0.00 & 0.00 & 0.17 & 0.00 & 0.00 \\
\hline Streptococcus & 0.12 & 0.00 & 0.17 & 0.00 & 0.17 \\
\hline Enterococcus & 0.12 & 0.00 & 0.00 & 0.00 & 0.00 \\
\hline Leuconostoc & 0.00 & 0.00 & 0.00 & 0.00 & 0.17 \\
\hline
\end{tabular}


Table S7B. Continued

\begin{tabular}{|c|c|c|c|c|c|}
\hline Bacillus & 0.00 & 0.00 & 0.17 & 0.00 & 0.00 \\
\hline Aeribacillus & 0.00 & 0.00 & 0.00 & 0.00 & 1.19 \\
\hline Paraliobacillus & 0.00 & 0.00 & 0.00 & 0.00 & 0.17 \\
\hline Turicibacter & 0.12 & 0.00 & 0.00 & 0.28 & 1.53 \\
\hline Catenibacterium & 0.00 & 0.17 & 0.17 & 0.00 & 0.00 \\
\hline Erysipelotrichaceae_incertae_sedis & 0.00 & 0.00 & 0.00 & 0.00 & 0.17 \\
\hline Allisonella & 0.00 & 0.00 & 0.35 & 0.00 & 0.00 \\
\hline Megasphaera & 0.00 & 0.00 & 0.17 & 0.28 & 0.00 \\
\hline Mitsuokella & 0.00 & 0.00 & 0.00 & 0.14 & 0.00 \\
\hline Microgenomates_genera_incertae_sedis & 0.12 & 0.00 & 0.00 & 0.00 & 0.00 \\
\hline Parcubacteria_genera_incertae_sedis & 0.12 & 0.00 & 0.00 & 0.00 & 0.51 \\
\hline Fibrobacter & 0.00 & 0.17 & 0.17 & 1.81 & 0.00 \\
\hline Armatimonas/Armatimonadetes_gpl & 0.00 & 0.17 & 0.00 & 0.00 & 0.00 \\
\hline Elusimicrobium & 0.00 & 0.00 & 0.17 & 0.00 & 0.00 \\
\hline Turneriella & 0.00 & 0.00 & 0.17 & 0.00 & 0.00 \\
\hline Victivallis & 0.00 & 0.00 & 0.00 & 0.14 & 0.00 \\
\hline unclassified_Planctomycetaceae & 3.95 & 4.21 & 2.09 & 0.00 & 0.00 \\
\hline unclassified_Rhodocyclaceae & 37.24 & 47.64 & 25.44 & 0.00 & 0.51 \\
\hline unclassified_Burkholderiales_incertae_sedis & 2.71 & 4.38 & 2.09 & 0.00 & 0.34 \\
\hline unclassified_Comamonadaceae & 2.59 & 4.55 & 4.18 & 6.12 & 6.29 \\
\hline unclassified_Burkholderiaceae & 0.00 & 0.17 & 0.00 & 0.00 & 0.85 \\
\hline unclassified_Oxalobacteraceae & 0.00 & 0.00 & 0.00 & 0.00 & 0.68 \\
\hline unclassified_Nitrosomonadaceae & 0.74 & 1.68 & 1.05 & 0.00 & 0.00 \\
\hline unclassified_Neisseriaceae & 0.00 & 0.00 & 0.17 & 0.14 & 0.00 \\
\hline unclassified_Enterobacteriaceae & 6.17 & 0.00 & 10.80 & 0.97 & 0.68 \\
\hline unclassified_Pseudomonadaceae & 0.00 & 0.00 & 0.17 & 0.00 & 1.19 \\
\hline unclassified_Moraxellaceae & 0.00 & 0.00 & 0.00 & 3.34 & 0.00 \\
\hline unclassified_Xanthomonadaceae & 0.12 & 0.17 & 0.35 & 0.00 & 0.00 \\
\hline unclassified_Succinivibrionaceae & 0.00 & 0.00 & 0.17 & 0.00 & 0.00 \\
\hline unclassified_Aeromonadaceae & 0.00 & 0.00 & 0.00 & 0.70 & 0.17 \\
\hline unclassified_Halomonadaceae & 0.00 & 0.00 & 0.00 & 0.00 & 1.19 \\
\hline unclassified_Bradyrhizobiaceae & 0.74 & 0.00 & 0.52 & 0.00 & 0.17 \\
\hline unclassified_Methylocystaceae & 0.00 & 0.17 & 0.00 & 0.00 & 0.00 \\
\hline unclassified_Phyllobacteriaceae & 0.00 & 0.00 & 0.17 & 0.00 & 0.34 \\
\hline unclassified_Rhodobacteraceae & 3.70 & 0.17 & 1.39 & 0.00 & 0.85 \\
\hline unclassified_Sphingomonadaceae & 0.62 & 0.34 & 0.17 & 0.00 & 0.17 \\
\hline unclassified_Acetobacteraceae & 0.12 & 0.00 & 0.17 & 0.00 & 0.00 \\
\hline unclassified_Caulobacteraceae & 0.00 & 0.00 & 0.00 & 0.00 & 0.51 \\
\hline unclassified_Polyangiaceae & 0.86 & 2.19 & 0.87 & 0.00 & 0.00 \\
\hline unclassified_Nannocystaceae & 0.62 & 0.17 & 0.35 & 0.00 & 0.00 \\
\hline unclassified_Cystobacteraceae & 0.12 & 0.00 & 0.17 & 0.00 & 0.00 \\
\hline
\end{tabular}


Table S7B. Continued

\begin{tabular}{|c|c|c|c|c|c|}
\hline unclassified_Desulfovibrionaceae & 0.00 & 0.00 & 0.00 & 0.14 & 0.00 \\
\hline unclassified_Anaerolineaceae & 1.97 & 3.70 & 3.83 & 0.00 & 0.00 \\
\hline unclassified_Caldilineaceae & 0.25 & 0.67 & 0.52 & 0.00 & 0.00 \\
\hline unclassified_Chloroflexaceae & 0.00 & 0.00 & 0.17 & 0.00 & 0.00 \\
\hline unclassified_"Leptotrichiaceae" & 0.00 & 0.17 & 0.00 & 0.83 & 0.17 \\
\hline unclassified_"Fusobacteriaceae" & 0.00 & 0.00 & 0.35 & 0.00 & 0.00 \\
\hline unclassified_Flavobacteriaceae & 0.12 & 0.17 & 0.17 & 0.56 & 0.85 \\
\hline unclassified_Chitinophagaceae & 0.62 & 0.00 & 0.35 & 0.00 & 0.00 \\
\hline unclassified_"Porphyromonadaceae" & 0.12 & 0.00 & 0.00 & 0.14 & 0.00 \\
\hline unclassified_"Prevotellaceae" & 0.00 & 0.00 & 0.87 & 40.89 & 0.00 \\
\hline unclassified_Acidimicrobiaceae & 0.00 & 0.00 & 0.17 & 0.00 & 0.00 \\
\hline unclassified_Intrasporangiaceae & 0.25 & 0.17 & 0.35 & 0.00 & 0.00 \\
\hline unclassified_Nocardioidaceae & 0.12 & 0.00 & 0.35 & 0.00 & 0.34 \\
\hline unclassified_Propionibacteriaceae & 0.00 & 0.00 & 0.00 & 0.14 & 0.00 \\
\hline unclassified_Micromonosporaceae & 0.00 & 0.00 & 0.00 & 0.00 & 0.17 \\
\hline unclassified_Coriobacteriaceae & 0.00 & 0.00 & 0.00 & 0.14 & 0.00 \\
\hline unclassified_Verrucomicrobiaceae & 0.12 & 0.17 & 0.35 & 0.00 & 0.00 \\
\hline unclassified_Opitutaceae & 0.12 & 0.17 & 0.00 & 0.00 & 0.00 \\
\hline unclassified_Lachnospiraceae & 0.25 & 0.00 & 0.35 & 4.73 & 0.34 \\
\hline unclassified_Clostridiaceae 1 & 0.37 & 0.17 & 0.52 & 0.70 & 9.86 \\
\hline unclassified_Peptostreptococcaceae & 0.49 & 0.51 & 0.87 & 0.28 & 3.40 \\
\hline unclassified_Ruminococcaceae & 0.25 & 0.34 & 0.00 & 0.97 & 0.68 \\
\hline unclassified_Bacillaceae 2 & 0.00 & 0.00 & 0.00 & 0.00 & 0.17 \\
\hline unclassified_Acidaminococcaceae & 0.00 & 0.00 & 0.17 & 0.56 & 0.00 \\
\hline unclassified_Veillonellaceae & 0.00 & 0.00 & 0.00 & 0.42 & 0.00 \\
\hline unclassified_Deinococcaceae & 0.00 & 0.00 & 0.00 & 0.00 & 0.17 \\
\hline unclassified_Spirochaetaceae & 0.00 & 0.00 & 0.17 & 0.00 & 0.00 \\
\hline
\end{tabular}

Apart from the similarity in containing more unclassified bacterial communities (on a genus level) than classified ones, the failed plants differ from successful wastewater treatment plants in the composition of their bacterial communities as well as the presence of unique bacterial species. Furthermore, more than 70 unique genera were only found in successful EBPR with relative abundance ranging from 2 to $6 \%$, while failed EBPR also revealed 69 unique genera at relative abundance ranging from 1 to $5.8 \%$ of the total microbial population (Fig. 5). It was noted that most of these unique genera from the successful EBPR belonged to Gammaproteobacteria, Betaproteobacteria, and Actinobacteria, while those from the failed EBPR belonged to Alphaproteobacteria, Betaproteobacteria, and Clostridia. The microbial composition of activated sludge has been seen as critical since they play a major role in the treatment of wastewater [21]. In order to enhance the wastewater treatment efficiency in removing phosphate, fermenter has been incorporated in the EBPR systems. The fermenter plays an important role in augmenting the availability of VFA needed by phosphate-accumulating organisms to ensure reliable phosphorus removal [5]. In the present study, fermenting bacterial genera such as Acidovorax (2.36\%), Enhydrobacter (1.25\%), Aeromonas (3.62\%), Tolumonas (1.81\%), Prevotella (7.09\%), Faecalibacterium (0.56\%), Fibrobacter (1.81\%), unclassified Comamonadaceae (6.12\%), unclassified Moraxellaceae (3.34\%), unclassified Prevotellaceae (40.89\%), and unclassified Lachnospiraceae $(4.73 \%)$ were found in higher abundance in the successful EBPR as compared to the failed EBPR (Table S7), and are seen as crucial for VFA production [36-37]. However, in the failed EBPR, Acinetobacter known to be part of the non-fermenting bacteria $[13,37]$ were also reported as the second most abundant genus in contrast to BWTW. In order to 
remove pollutants using conventional EBPR processes, activated sludge is cycled under sequential anaerobic, anoxic, and aerobic conditions that promote the growth of specific microorganisms respective of each condition [10]. Under anaerobic conditions, the present study has shown that the bacterial community of successful EBPR was predominantly containing phosphate-accumulating bacteria such as unclassified Rhodocyclaceae (47.64\%) $[8,38]$, followed by glycogen-accumulating organisms (GAOs) such as Propionivibrio (8.59\%) [39]. Even though many EBPR wastewater treatment plants run well, the deterioration of their pollutant removal capacity due to the competition between beneficial (PAOs) and detrimental (GAOs) organisms have also been reported [5, 33]. It was further observed that the anaerobic zone of the successful EBPR also had high abundance of an additional bacterial community responsible for nitrification and denitrification, such as unclassified Comamonadaceae (4.55), unclassified Burkholderiales incertae sedis (4.38), Planctomyces (1.68), Nitrospira (1.68), unclassified Nitrosomonadaceae (1.68), unclassified Anaerolineaceae (3.7), and so on $[3,7,33]$. In contrast, despite the similarity
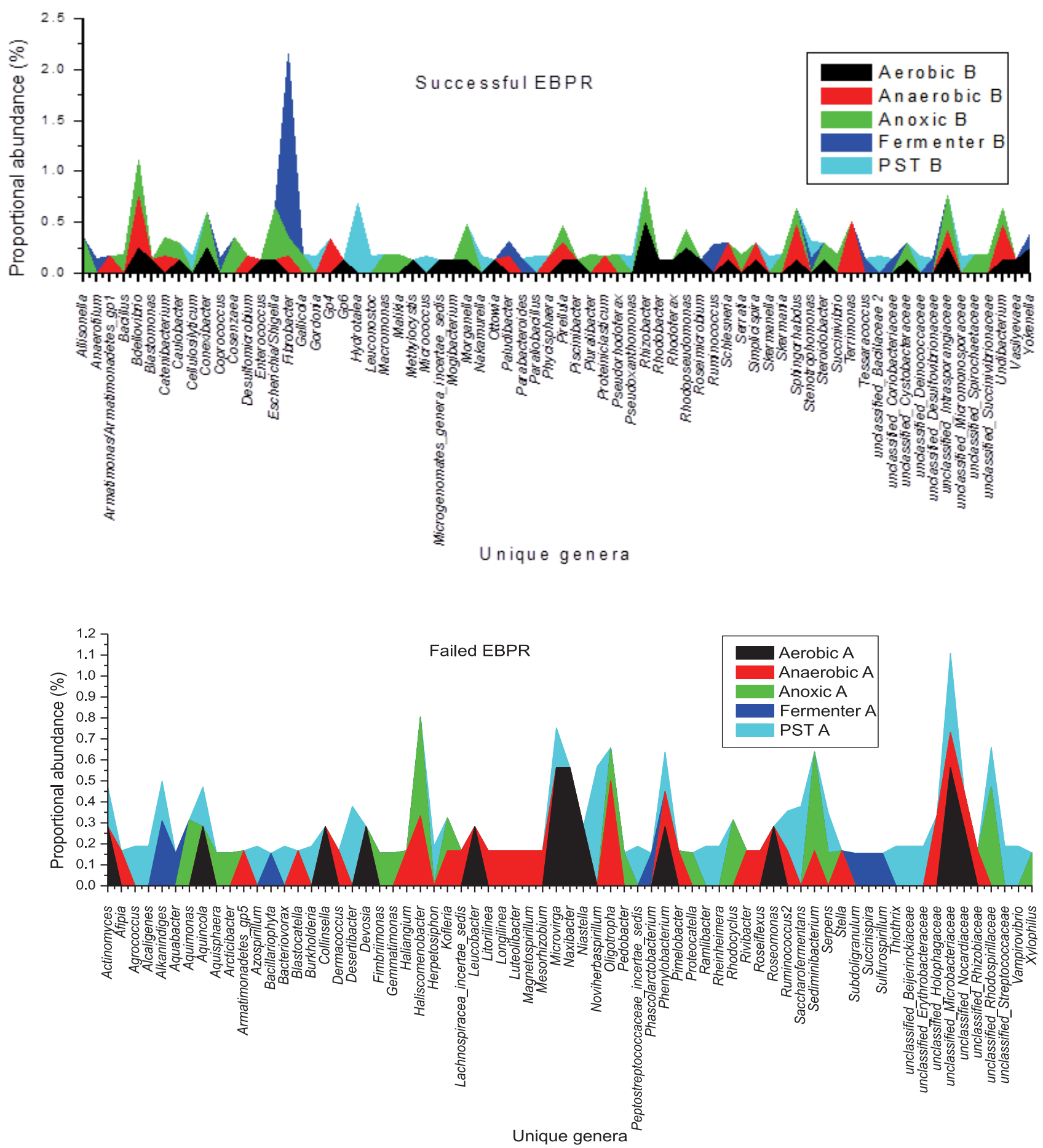

Fig. 5. Relative abundance of unique genera from failed EBPR and successful EBPR wastewater treatment plants. 
of bacterial communities between anaerobic zones of both plants, the abundance of phosphate-accumulating, -nitrifying and -denitrifying bacteria at the failed EBPR were found to be very low compared to the successful EBPR. Sidat et al. [40] reported that the presence of P-removing bacteria under anaerobic conditions is due to the fact that they are needed to convert VFAs synthesised in the zone by fermenters to polyhydroxybutyrate (PHB), which is stored intracellularly. Under anoxic conditions, a significant decrease of unclassified Rhodocyclaceae $(25.44 \%$ - over $50 \%$ compared to their abundance in the anaerobic zone) was noted, while a drastic increase of unclassified Enterobacteriaceae $(10.8 \%$ - considered a special species due to their ability to remove both nitrate and phosphate under anoxic conditions) was also observed in the successful EBPR [3-4]. Furthermore, a large number of nitrifying/denitrifying bacteria such as Nitrospira (5.4\%), Proteus (4.88\%), unclassified Comamonadaceae (4.18\%), unclassified Anaerolineaceae (3.83\%), Clostridium XI (2.44\%), Arcobacter (2.09\%), Tetrasphaera $(2.09 \%)$, unclassified Planctomycetaceae (2.09\%), unclassified Burkholderiales incertae sedis (2.09\%), unclassified Rhodobacteraceae (1.39\%), Nannocystis (1.22\%), Caenimonas (1.05\%), and unclassified Nitrosomonadaceae $(1.05 \%)$ was also noted. In contrary to the successful EBPR, the failed EBPR showed an increase in abundance of unclassified Rhodocyclaceae (35.79\%), unclassified Anaerolineaceae (9.26\%), and Propionivibrio (5.49\%) when compared to anaerobic and anoxic zones. In addition, the anoxic zone of the failed EBPR also showed a diverse nitrifying and denitrifying bacterial community despite the fact that there was less abundance compared to the anoxic zone of the successful EBPR. It has been proven that anaerobic and anoxic conditions are designed to promote nitrification and denitrification, respectively [5].

Under subsequent aerobic conditions, the present study revealed that both plants had a significant difference in terms of predominant microbial genera. The successful EBPR was found to have high relative abundance of PAOs with unclassified Rhodocyclaceae (37.24\%) as the most dominant one, followed by Proteus $(6.41 \%)$. In the failed EBPR however, Acidovorax (26.2\%) was reported to be the most abundant genera, followed by unclassified Comamonadaceae (13.52\%) and Brevundimonas (6.76\%). Despite the fact that Acidovorax is not so common in the removal of phosphate, Ren et al. [41] reported that this genus can participate in the removal of both phosphate and nitrite. Gonzalez-Gil and Holliger [42] further reported that most of the species belonging to Acidovorax are capable of heterotrophic denitrification of nitrate and may utilise acetate, propionate, and PHB from decomposing cells as the carbon source for denitrification. However, this was in disagreement with Siezen and Galardini [43], who reported that species belonging to Acidovorax are mostly responsible for biofilm formation and flocculation. The second most abundant genus (Comamonadaceae) at the failed EBPR has also been reported by Ong et al. [44] as being strictly aerobic, non-fermentative, and capable of accumulating PHB. Moreover, the present study also revealed the presence of nitrifying and denitrifying bacteria in the aerobic zone (Table S7). Fu et al. [45] reported that a denitrifier's ability to grow in aerobic conditions is probably attributed to the variation of DO concentration gradient in the biofilm, creating the anoxic micro-environment in the deeper layer of biofilm, which is beneficial for the growth of denitrifiers. Bai et al. [3] also stated that the coexistence of nitrifying and denitrifying bacteria in the aerobic zone is beneficial as it facilitates simultaneous nitrification and denitrification occurring in the zone. In the present study, most of the dominant microorganisms recovered have been reported previously for their involvement in the removal of phosphate and nitrate from wastewater [7, 46-48]. Furthermore, the microbial pattern noted in the present study is also similar to previous observations [4, 41, 49]. It should be mentioned that not many studies have been carried out to investigate microbial ecology from the fullscale EBPR. In addition, this study corroborates with the findings by Zhang et al. [31] in reporting some rare bacterial classes: Acidobacteria Gp3, Acidobacteria Gp6, and Acidobacteria Gp4. However, the study disagrees with the findings of Martín et al. [50] and Albertsen et al. [4] by revealing that PAOs populating the selected EBPRs were closely related to Rhodocyclus bacteria and Actinobacteria instead of Candidatus Accumulibacter phosphatis. The results showed that most of the samples from failed and successful EBPRs had almost the same composition as they were clustered together with Jaccard values ranging from 65 to $100 \%$. These results were confirmed by the rarefaction curves that showed little to no existing dissimilarity between treatment zones of both plants with the exception of the aerobic zone. However, Lawson et al. [32] also revealed low dissimilarity between the bioreactor representing the lab-scale EBPR.

\section{Environmental Variables}

The results of the physicochemical parameters of wastewater samples collected from different zones (PST, fermenter, anaerobic, anoxic, and aerobic) of the failed and successful EBPR treatment plants are shown in Table S8. As a phosphorus removal plant, the results revealed a decrease in phosphorus content from $33.35 \mathrm{mg} \mathrm{L}^{-1}$ to $0.97 \mathrm{mg} \mathrm{L}^{-1}$, and $18.25 \mathrm{mg} \mathrm{L}^{-1}$ to $1.91 \mathrm{mg} \mathrm{L}^{-1}$ from the successful and failed EBPRs, respectively, as wastewater was subjected to anaerobic and aerobic treatment from EBPRs. A similar observation was noted with COD, which decreased from both EBPRs. The collected wastewater samples appeared to be neutral to slightly alkaline (Table S8). However, temperatures ranged $22.2-23.5^{\circ} \mathrm{C}$ for the successful EBPR and $22.2-23.4^{\circ} \mathrm{C}$ for the failed EBPR, showing that both reactors had similar temperature conditions.

DO depletion from aerobic to anaerobic zones highlighted the good performance of the treatment plants. Electrical conductivity (EC) as salinity has been reported as a significant parameter in the bacterial community as 
Table S8: Environmental variables of the wastewater samples

\begin{tabular}{|c|c|c|c|c|c|c|}
\hline & Parameters & PST & Fermenter & Anaerobic & Anoxic & Aerobic \\
\hline \multirow{7}{*}{$\begin{array}{l}\text { Successful } \\
\text { EBPR }\end{array}$} & Phosphate(mg L-1) & $33.35 \pm 4.2$ & $22.13 \pm 1.02$ & $26.38 \pm 2.93$ & $16.89 \pm 0.97$ & $0.97 \pm 0.01$ \\
\hline & Nitrate $\left(\mathrm{mg} \mathrm{L}^{-1}\right)$ & $3.23 \pm 0.89$ & $5.20 \pm 0.75$ & $3.17 \pm 1.34$ & $3.87 \pm 0.83$ & $3.07 \pm 0.07$ \\
\hline & $\mathrm{COD}\left(\mathrm{mg} \mathrm{L}^{-1}\right)$ & $198.50 \pm 25.47$ & $164.33 \pm 7.97$ & $60.17 \pm 7.45$ & $43.95 \pm 2.84$ & $27.67 \pm 0.98$ \\
\hline & $\mathrm{EC}\left(\mu \mathrm{S} \mathrm{cm}^{-1}\right)$ & $634.00 \pm 94.15$ & $639.00 \pm 34.75$ & $827.00 \pm 41.009$ & $606.00 \pm 19.65$ & $589.00 \pm 47.81$ \\
\hline & Temperature $\left({ }^{\circ} \mathrm{C}\right)$ & $23.00 \pm 2.92$ & $22.20 \pm 2.94$ & $22.80 \pm 2.84$ & $22.60 \pm 2.89$ & $23.50 \pm 3.67$ \\
\hline & $\mathrm{DO}\left(\mathrm{mg} \mathrm{L}^{-1}\right)$ & $6.10 \pm 1.71$ & $1.64 \pm 0.92$ & $0.09 \pm 0.01$ & $0.27 \pm 0.02$ & $2.36 \pm 0.05$ \\
\hline & $\mathrm{pH}$ & $7.21 \pm 0.23$ & $7.08 \pm 1.54$ & $7.00 \pm 1.62$ & $6.99 \pm 0.95$ & $7.27 \pm 0.07$ \\
\hline \multirow{7}{*}{ Failed EBPR } & Phosphate(mg L-1) & $18.25 \pm 2.27$ & $25.63 \pm 5.03$ & $26.65 \pm 2.64$ & $18.61 \pm 2.84$ & $1.91 \pm 0.01$ \\
\hline & Nitrate $\left(\mathrm{mg} \mathrm{L}^{-1}\right)$ & $3.79 \pm 0.21$ & $3.19 \pm 1.84$ & $2.01 \pm 0.81$ & $3.35 \pm 0.92$ & $4.11 \pm 0.03$ \\
\hline & $\mathrm{COD}\left(\mathrm{mg} \mathrm{L}^{-1}\right)$ & $225.17 \pm 17.07$ & $181.83 \pm 43.54$ & $96.83 \pm 17.94$ & $18.50 \pm 1.67$ & $35.17 \pm 2.74$ \\
\hline & $\mathrm{EC}\left(\mu \mathrm{S} \mathrm{cm}^{-1}\right)$ & $655.00 \pm 26.93$ & $664.00 \pm 39.65$ & $947.00 \pm 25.84$ & $753.00 \pm 52.93$ & $755.00 \pm 21.93$ \\
\hline & Temperature $\left({ }^{\circ} \mathrm{C}\right)$ & $23.00 \pm 1.98$ & $22.20 \pm 3.29$ & $22.80 \pm 3.91$ & $23.40 \pm 2.91$ & $22.30 \pm 1.95$ \\
\hline & $\mathrm{DO}\left(\mathrm{mg} \mathrm{L}^{-1}\right)$ & $3.95 \pm 0.98$ & $1.65 \pm 0.10$ & $0.02 \pm 0.00$ & $0.38 \pm 0.03$ & $1.09 \pm 0.01$ \\
\hline & $\mathrm{pH}$ & $7.00 \pm 1.69$ & $7.01 \pm 0.81$ & $6.98 \pm 0.08$ & $7.23 \pm 0.92$ & $7.08 \pm 0.02$ \\
\hline
\end{tabular}

it regulates their composition and diversity [13, 51-52]. Similar to EC, $\mathrm{pH}$ was also seen as an important factor with temperature and DO regulating the overall diversity and composition level of bacteria in aquatic environments [13]. Based on the variance inflation factors, Wang and co-authors [13] revealed that $\mathrm{COD}$, total nitrogen, total phosphorus, $\mathrm{DO}, \mathrm{pH}$, and temperature variations were relatively correlated to the bacterial communities. In their study investigating the impact of temperature on microbial communities, Siggins et al. [53] also revealed

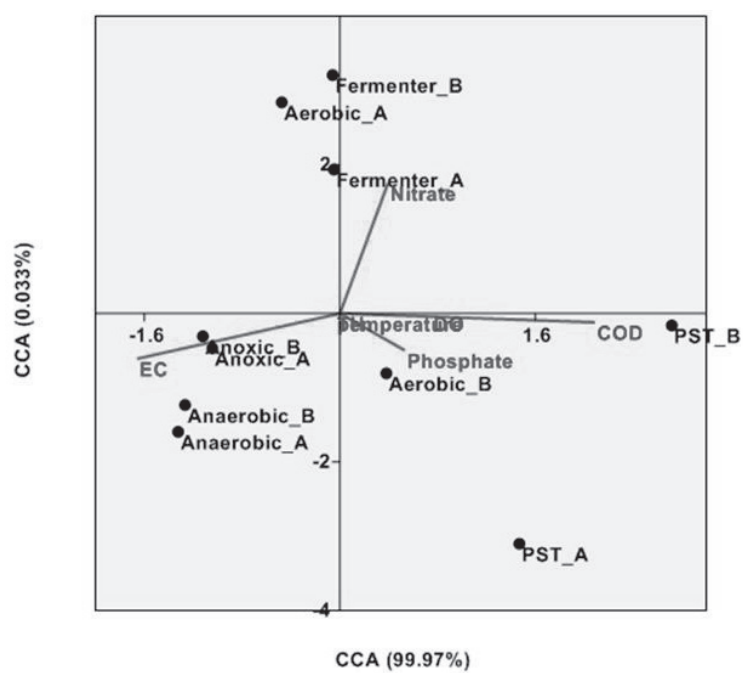

Fig. 6. Canonical correspondence analysis (CCA) comparing the variation of environmental parameters (EC: electrical conductivity) from each sampling point (PST, fermenter, anaerobic, anoxic, and aerobic) and their possible effects on a bacterial community. The CCA first axis (99.97\%) and the second axis $(0.033 \%)$ show the observed variation between parameters. that temperature is one of the most important parameters influencing bacterial composition in the environment. This was also revealed by the present finding when performing the canonical correspondence analysis (CCA) to assess the correlation between environmental factors and bacterial communities (Fig. 6). The CCA diagram displayed $99.97 \%$ of the variance in the bacteriaenvironmental relationships at the first axis and $0.033 \%$ of the variance at the second axis. However, the relationship between environmental factors and bacterial shift (pyrosequencing data) was significant at both the first and the second axis $(\mathrm{p}<0.05)$. Environmental factors - namely $\mathrm{COD}, \mathrm{DO}, \mathrm{pH}$, temperature, and $\mathrm{PO}_{4}^{-3}$ - showed a strong positive correlation with the first axis and a negative correlation with the second axis, while $\mathrm{NO}_{3}^{-}$showed a strong positive correlation with both first and second axes. Electrical conductivity showed a strong negative correlation with both the first and second axes. While comparing EBPR zones, anoxic as well as anaerobic zones from both successful and failed EBPRs were primarily linked to EC, while fermenters linked to nitrate. Not surprisingly, the aerobic zone from the successful EBPR appeared to be linked to $\mathrm{COD}, \mathrm{DO}, \mathrm{pH}$, temperature, and $\mathrm{PO}_{4}^{-3}$ load, while the aerobic zone from the failed EBPR was only linked to nitrate load, which highlighted the possible dissimilarity. Furthermore, temperature and $\mathrm{pH}$ appeared to have low or no significant impact on bacterial community structures in both EBPRs despite being positively correlated with the first axis and ne-gatively correlated with the second. This study is in agreement with the findings of Wang et al. [10], who reported the possible links between COD, DO, and temperature with microbial community functional structures in EBPRs. 


\section{Conclusion}

This study investigated the bacterial community of two full-scale EBPR wastewater treatment plants using a pyrosequencing platform. A comparison of the bacterial diversity of the two full-scale EBPRs provided links between the bacterial community structures from both successful and failed EBPRs. It was observed that Proteobacteria was the most predominant phylum of both EBPRs within each sampling zone, except in fermenters, where Bacteriodetes was the dominant one. Apart from the 18 phyla shared between both EBPRs, two unique phyla were noted for successful EBPR (Chlamydiae and Gemmatimonadetes) and failed EBPR (Microgenomates and Fibrobacteres). In the present study the constant failure of Bushkoppies was noted to be due to less abundance of fermenting bacteria from the fermenter and less abundance of nitrifying/denitrifying as well as phosphate-accumulating bacteria in the bioreactor. Furthermore, the present study suggested that the high abundance of Acidovorax in the Bushkoppies aerobic zone could have also been the cause of the constant failure of this EBPR. The study also showed a relatively high abundance of detrimental bacteria such as GAOs throughout all zones of this bioreactor when compared to the successful EBPR. However, bacterial communities from both EBPRs showed a similar correlation with EC in fermenters and anaerobic zones, while temperature and $\mathrm{pH}$ showed a similar correlation throughout all the EBPRs. The difference between the successful and failed EBPRs was most apparent in the aerobic zones, as this zone from both EBPR was linked with different environmental factors.

\section{Authors' Contributions}

IK Conceived and designed the experiments; IK MT Contributed reagents/materials/analysis tools; IK carried out the molecular genetic studies, participated in the sequence alignment and drafted the manuscript; and IK SA MT critically reviewed the manuscript. The three authors read and approved the final manuscript.

\section{Acknowledgements}

The authors are grateful to the National Research Foundation (NRF) for funding this project (grant No. 103907).

\section{References}

1. KAMIKA I., MOMBA M.N.B. Comparing the tolerance limit of selected bacterial and protozoan species to nickel in wastewater systems. Sci. Total Environ. 400, 172, 2011.

2. TEKLEHAIMANOT G.Z., KAMIKA I., COETZEE M.A.A., MOMBA M.N.B. Population growth and its impact on the design capacity and performance of the wastewater treatment plants in Sedibeng and Soshanguve, South Africa. Environ Manag. 56 (4), 984, 2015.

3. BAI Y., ZHANG Y., QUAN X., CHEN S. Nutrient removal performance and microbial characteristics of a full-scale IFAS-EBPR process treating municipal wastewater. Water Sci Technol. 73 (6), 1261, 2016.

4. ALBERTSEN M., HANSEN L.B.S., SAUNDERS A.M.S., NIELSEN P.H., NIELSEN K.L. A metagenome of a full-scale microbial community carrying out enhanced biological phosphorus removal. ISME J 6, 1094, 2012.

5. KAMIKA I., COETZEE M., MAMBA B.B., MSAGATI T., MOMBA M.N.B. The Impact of Microbial Ecology and Chemical Profile on the Enhanced Biological Phosphorus Removal (EBPR) Process: A Case Study of Northern Wastewater Treatment Works, Johannesburg. Int. J. Environ. Res. Public Health 11, 2876, 2014.

6. CLOETE T.E., STEYN P.L. A combined fluorescent antibody membrane filter technique for enumerating Acinetobacter in activated sludges. In Biological phosphate removal from wastewater; Ramadori R. Ed., Pergamon: Oxford, UK, 335, 1987.

7. SEVIOUR R.J., MINO T., ONUKI M. The microbiology of biological phosphorus removal in activated sludge systems. FEMS Microbiol. Rev. 27, 99, 2003.

8. BOND P.L., HUGENHOLTZ P., KELLER J., BLACKALL L.L. Bacterial community structures of phosphateremoving and non-phosphate-removing activated sludges from sequencing batch reactors. Appl. Environ. Microbiol. 61, 1910, 1995.

9. NGUYEN H.T.T., LE V.Q., HANSEN A.A., NIELSEN J.L., NIELSEN P.H. High diversity and abundance of putative polyphosphate-accumulating Tetrasphaera-related bacteria in activated sludge systems. FEMS Microbiol Ecol. 76, 256, 2011.

10. WANG X., XIA Y., WEN X., YANG Y., ZHOU J. Microbial Community Functional Structures in Wastewater Treatment Plants as Characterized by GeoChip. PLoS One 9 (3), e93422, 2014. doi:10.1371/journal.pone.0093422

11. KUNIN V., COPELAND A., LAPIDUS A., MAVROMATIS K., HUGENHOLTZ P. A bioinformatician's guide to metagenomics. Microbiol Mol Biol Rev. 72, 557, 2008.

12. BROWN M.V., PHILIP G.K., BUNGE J.A., SMITH M.C., BISSETT A., LAURO F.M., FUHRMAN J.A., DONACHIE S.P. Microbial community structure in the North Pacific Ocean. ISME J. 3, 1374, 2009.

13. WANG X., HU M., XIA Y., WEN X., DING K. Pyrosequencing Analysis of Bacterial Diversity in 14 Wastewater Treatment Systems in China. Appl. Environ. Microbiol. 78 (19), 7042, 2012.

14. MONTEIROM.I.C., FERREIRA F.N., OLIVEIRAN.M.M., AVILA A.K. Simplified version of the sodium salicylate method for nitrate analysis in drinking waters. Anal. Chim. Acta. 477 (1), 125, 2003.

15. APHA. Standard Methods for the Examination of Water and Wastewater, $20^{\text {th }}$ ed.; American Public Health Association: Washington, DC, 2001.

16. SCHLOSS P.D., WESTCOTT S.L., RYABIN T., HALL J.R., HARTMANN M., HOLLISTER E.B., LESNIEWSKI R.A., OAKLEY B.B., PARKS D.H., ROBINSON C.J., SAHL J.W., STRES B., THALLINGER G.G., VAN HORN D.J., WEBER C.F. Introducing mothur: OpenSource, Platform-Independent, Community-Supported Software for Describing and Comparing Microbial Communities. Appl. Environ. Microbiol. 75 (23), 7537, 2009. 
17. EDGAR R.C., HAAS B.J., CLEMENTE J.C., QUINCE C., KNIGHT R. UCHIME improves sensitivity and speed of chimera detection. Bioinformatics 27 (16), 2194, 2011.

18. COLE J.R., WANG Q., FISH J.A., CHAI B., MCGARRELL D.M., SUN Y., BROWN C.T., PORRAS-ALFARO A., KUSKE. C.R., TIEDJE J.M. Ribosomal Database Project: data and tools for high throughput rRNA analysis. Nucl. Acids Res. 42 (Database issue) D633, 2014.

19. HAMMER Ø.,HARPERD.A.T., RYAN P.D. Paleontological statistics software package for education and data analysis. Palaeontol Electronica 4 (1), 9, 2001.

20. YE L., ZHANG T. Bacterial communities in different sections of a municipal wastewater treatment revealed by $16 \mathrm{~S}$ rDNA pyrosequencing. Appl Microbiol Biotechnol 97 (6), 2681, 2013.

21. LV X.M., SHAO M.F., LI C.L., GAO X.L., SUN F.Y. A comparative study of the bacterial community in denitrifying and traditional enhanced biological phosphorus removal processes. Microbes Environ. 29 (3), 261, 2014.

22. ZHOU J., HE Z., YANG Y., DENG Y., TRINGE S.C., ALVAREZ-COHEN L. High-throughput metagenomic technologies for complex microbial community analysis: open and closed formats. mBio 6 (1), e02288-14, 2015. doi:10.1128/mBio.02288-14.

23. OULAS A., PAVLOUDI C., POLYMENAKOU P., PAVLOPOULOS G.A. PAPANIKOLAOU N., KOTOULAS G., ARVANITIDIS C., LLIOPOULOS L. Metagenomics: Tools and Insights for Analyzing NextGeneration Sequencing Data Derived from Biodiversity Studies. Bioinform Biol Insights. 9, 75, 2015.

24. HAAS B.J., GEVERS D., EARL A.M., FELDGARDEN M., WARD D.V., GIANNOUKOS G., CIULLA D., TABBAA D., HIGHLANDER S.K. SODERGEN E., METHE B., DESANTIS T.Z., THE HUMAN MICROBIOME CONSORTIUM, PETROSINO J.F., KNIGHT R., BIRREN B.W. Chimeric 16S rRNA sequence formation and detection in Sanger and 454-pyrosequenced PCR amplicons. Genome Res. 21 (3), 494, 2011.

25. NIELSEN P.H., MIELCZAREK A.T., KRAGELUND C., NIELSEN J.L., SAUNDERS A.M., KONG Y., HANSEN A.A., VOLLERTSEN J. A conceptual ecosystem model of microbial communities in enhanced biological phosphorus removal plants. Water Res. 44 (17), 5070, 2010.

26. GIRVAN M.S., CAMPBELL C.D., KILLHAM K., PROSSER J.I., GLOVER L.A. Bacterial diversity promotes community stability and functional resilience after perturbation. Environ Microbiol 7, 301, 2005.

27. SAIKALY P.E., OERTHER D.B. Diversity of dominant bacterial taxa in activated sludge promotes functional resistance following toxic shock loading. Microb Ecol 61, $557,2011$.

28. RASHEED Z., RANGWALA H., BARBARÁ D. 16S rRNA metagenome clustering and diversity estimation using locality sensitive hashing. BMC Syst Biol 7, (4), S11, 2013.

29. BENTO F.M., DE OLIVEIRA CAMARGO F.A., OKEKE B.C., FRANKENBERGER JR W.T. Diversity of biosurfactant producing microorganisms isolated from soils contaminated with diesel oil. Microbiol Res 160 (3), 249, 2005.

30. LEPRIEUR F., ALBOUY C., DE BORTOLI J., COWMAN P.F., BELLWOOD D.R., MOUILLOT D. Quantifying Phylogenetic Beta Diversity: Distinguishing between 'True' Turnover of Lineages and Phylogenetic Diversity Gradients. PLoS One 7 (8), e42760, 2012.
31. ZHANG T., SHAO M.F., YE L. 454 pyrosequencing reveals bacterial diversity of activated sludge from 14 sewage treatment plants. ISME J. 6 (6), 1137, 2012.

32. LAWSON C.E., STRACHAN B.J., HANSON N.W., HAHN A.S., HALL E.R., RABINOWITZ B., MAVINIC D.S., RAMEY W.D., HALLAM S.J. Rare taxa have potential to make metabolic contributions in enhanced biological phosphorus removal ecosystems. Environ. Microbiol. 17 (12), 4979, 2015.

33. TIAN M., ZHAO F., SHEN X., CHU K., WANG J., CHEN S., GUO Y., LIU H. The first metagenome of activated sludge from full-scale anaerobic/anoxic/oxic (A2O) nitrogen and phosphorus removal reactor using Illumina sequencing. J. Environ. Sci. 181, 2015.

34. JIN H., LI B., PENG X., CHEN L. Metagenomic analyses reveal phylogenetic diversity of carboxypeptidase gene sequences in activated sludge of a wastewater treatment plant in Shanghai, China. Ann Microbiol. 64 (2), 689, 2014.

35. FENG C.J., ZHANG Z.J., WANG S.M., FANG F., YE Z.Y., CHEN S.H. Characterization of microbial community structure in a hybrid biofilm-activated sludge reactor for simultaneous nitrogen and phosphorus removal. J. Environ. Biol. 34, 489, 2013

36. MEEHAN C.J., BEIKO R.G. A Phylogenomic View of Ecological Specialization in the Lachnospiraceae, a Family of Digestive Tract-Associated Bacteria. Genome Biol Evol. 6 (3), 703, 2014.

37. RANSOM-JONES E., JONES D.L., MCCARTHY A.J., MCDONALD J.E. The fibrobacteres: an important phylum of cellulose-degrading bacteria. Microbial Ecol 63, 267, 2012.

38. CHRISTENSSON M., BLACKALL L.L., WELANDER T. Metabolic transformations and characterization of the sludge community in an enhanced biological removal system. Appl. Microbiol. Biotechnol. 49, 226, 1998.

39. ALBERTSEN M., MCLLROY S.J., STOKHOLMBJERREGAARD M., KARST S.M., NIELSEN P.H. "Candidatus Propionivibrio aalborgensis": A Novel Glycogen Accumulating Organism Abundant in Full-Scale Enhanced Biological Phosphorus Removal Plants. Front Microbiol. 7, 1033, 2016. DOI: 10.3389/fmicb.2016.01033

40. SIDAT M., BUX F., KASAN H.C. Polyphosphate accumulation by bacteria isolated from activated sludge. Water SA 25 (2), 175, 1999.

41. REN Y., WEI C.H., XIAO K.J. Characterisation of microorganisms responsible for EBPR in a sequencing batch reactor by using the $16 \mathrm{~S}$ rDNA-DGGE method. Water SA 33, 123, 2007.

42. GONZALEZ-GIL G., HOLLIGER C. Dynamics of microbial community structure of and enhanced biological phosphorus removal by aerobic granules cultivated on propionate or acetate. Appl. Environ. Microbiol. 77 (22), 8041, 2011.

43. SIEZEN R.J., GALARDINI M. Genomics of biological wastewater treatment. Microbial Biotechnol. 1 (5), 333, 2008.

44. ONG Y.H., CHUA A.S.M., HUANG Y.T., NGOH G.C., YOU S.J. The microbial community in a high-temperature enhanced biological phosphorus removal (EBPR) process. Sustainable Environ Res. 26 (1), 14, 2016.

45. FU B., LIAO X.Y., DING L.L., REN H.Q. Characterization of microbial community in an aerobic moving bed biofilm reactor applied for simultaneous nitrification and denitrification. World J. Microbiol. Biotechnol. 26 (11), 1981, 2010. 
46. KONG Y., ONG S.L. NG W.J., LIU W.T. Diversity and distribution of a deeply branched novel proteobacterial group found in anaerobic - aerobic activated sludge processes. Environ. Microbiol. 4 (11), 753, 2002.

47. MINO T. Microbial Selection of PolyphosphateAccumulating Bacteria in Activated Sludge Wastewater Treatment Processes for Enhanced Biological Phosphate Removal. Biochemistry Mosc. 65 (3), 341, 2000.

48. RASHED E.M., MASSOUD M. The effect of effective microorganisms (EM) on EBPR in modified contact stabilization system. HBRC J. 11 (3), 384, 2014.

49. MULLAN A., QUINN J.P., MCGRATH J.W. Enhanced phosphate uptake and polyphosphate accumulation in Burkholderia cepacia grown under low-pH conditions. Microbial Ecol. 44, 69, 2002.

50. MARTÍN H.G., IVANOVA N., KUNIN V., WARNECKE F., BARRY K.W., MCHARDY A.C., YEATES C., HE
S., SALAMOV A.A., SZETO E., DALIN E., PUTNAM N.H., SHAPIRO H.J., PANGILINAN J.L., RIGOUTSOS I., KYRPIDES N.C., BLACKALL L.L., MCMAHON K.D., HUGENHOLTZ P. Metagenomic analysis of two enhanced biological phosphorus removal (EBPR) sludge communities. Nature Biotechnol. 24, 1263, 2006.

51. LOZUPONE C.A., KNIGHT R. Global patterns in bacterial diversity. Proc. Natl. Acad. Sci. USA 104, 11436, 2007.

52. MOHAMED D.J., MARTINY J.B.H. Patterns of fungal diversity and composition along a salinity gradient. ISME J. 5, 379, 2011.

53. SIGGINS A., ENRIGHT A.-M., O'FLAHERTY V. Temperature dependent $\left(3715^{\circ} \mathrm{C}\right)$ anaerobic digestion of a trichloroethylene-contaminated wastewater. Bioresour. Technol. 102 (17), 7645, 2011. 